\title{
HEAT TRANSFER ANALYSIS ON ROTATING FLOW \\ OF A SECOND-GRADE FLUID PAST A POROUS PLATE WITH VARIABLE SUCTION
}

\author{
T. HAYAT, ZAHEER ABBAS, AND S. ASGHAR
}

Received 2 November 2004

We deal with the study of momentum and heat transfer characteristics in a second-grade rotating flow past a porous plate. The analysis is performed when the suction velocity normal to the plate, as well as the external flow velocity, varies periodically with time. The plate is assumed at a higher temperature than the fluid. Analytic solutions for velocity, skin friction, and temperature are derived. The effects of various parameters of physical interest on the velocity, skin friction, and temperature are shown and discussed in detail.

\section{Introduction}

The study of non-Newtonian fluids has attracted much attention, because of their practical applications in engineering and industry particularly in extraction of crude oil from petroleum products, food processing, and construction engineering. Due to complexity of fluids, various models have been proposed. The equations of motion of nonNewtonian fluids are highly nonlinear and one order higher than the Navier-Stokes equations. Finding accurate analytic solutions to such equations is not easy. There is a particular class of non-Newtonian fluids namely the second-grade fluids for which one can reasonably hope to obtain an analytic solution. Important studies of second-grade fluids in various contexts have been given in the references $[1,3,6,7,9,10,11,12,13,17,19$, $20,21,22,24]$.

Since the pioneering work of Lighthill [16] there has been a considerable amount of research undertaken on the time-dependent flow problems dealing with the response of the boundary layer to external unsteady fluctuations about a mean value. Important contributions to the topic with constant and variable suction include the work of Stuart [25], Messiha [18], Kelley [15], Soundalgekar and Puri [23], and Hayat et al. [8].

Despite the above studies, no attention has been given to the study of the simultaneous effects of the rotation and heat transfer characteristics on the non-Newtonian flow with variable suction. Such work seems to be important and useful for gaining our basic understanding of such flow and partly for possible applications to geophysical and astrophysical problems. Also, heat transfer plays an important role during the handling and processing of non-Newtonian fluids. The understanding of heat transfer in boundary 
layer flows of non-Newtonian fluids is of importance in many engineering applications such as the design of thrust bearings and radial diffusers, transpiration cooling, drag reduction, thermal recovery of oils, and so forth. The primary purpose of the present paper is to make an investigation of the combined effects of rotation, and heat transfer characteristics on the flow of a second-grade fluid past a porous plate with variable suction. This work is concerned with a boundary value problem in a rotating flow. The analytical solution of the velocity field, skin-friction, and temperature distribution is obtained. Special attention is given to finding the analytical solutions and to describe the physical nature. Finally, in order to see the variations of different emerging parameters, the graphs are sketched and discussed.

\section{Mathematical formulation}

Let us consider an incompressible second-grade fluid past a porous plate. The plate and the fluid rotate in unison with an angular velocity $\Omega$ about the $z^{\prime}$-axis normal to the plate. The plate is located at $z^{\prime}=0$ having temperature $T_{0}$. The flow far away from the plate is uniform and temperature of the fluid is $T_{\infty}$.

For the problem under question, we consider the velocity and temperature fields as

$$
\begin{gathered}
\mathbf{V}=\left(u^{\prime}\left(z^{\prime}, t^{\prime}\right), v^{\prime}\left(z^{\prime}, t^{\prime}\right), w^{\prime}\left(z^{\prime}, t^{\prime}\right)\right), \\
T=T\left(z^{\prime}, t^{\prime}\right),
\end{gathered}
$$

in which $u^{\prime}, v^{\prime}$, and $w^{\prime}$ are the velocity components in $x^{\prime}, y^{\prime}$, and $z^{\prime}$ directions, respectively, and $T$ indicates the temperature.

The governing equations in absence of body forces and radiant heating are

$$
\begin{gathered}
\operatorname{div} \mathbf{V}=0, \\
\rho^{\prime}\left[\frac{d \mathbf{V}}{d t^{\prime}}+2 \mathbf{\Omega} \times \mathbf{V}+\mathbf{\Omega} \times(\boldsymbol{\Omega} \times \mathbf{r})\right]=\operatorname{div} \mathbf{T}, \\
\rho^{\prime} \frac{d e}{d t^{\prime}}=\mathbf{T} \cdot \mathbf{L}-\operatorname{div} \mathbf{q} .
\end{gathered}
$$

In above equations $d / d t^{\prime}, \rho^{\prime}, e, \mathbf{L}$, and $\mathbf{q}$ are, respectively, the material derivative, density, the specific internal energy, the gradient of velocity, the heat flux vector, and the radial distance $r^{2}=x^{2}+y^{2}$. The Cauchy stress $\mathbf{T}$ in an incompressible homogeneous fluid of second grade is of the form

$$
\begin{gathered}
\mathbf{T}=-p \mathbf{I}+\mu \mathbf{A}_{1}+\alpha_{1} \mathbf{A}_{2}+\alpha_{2} \mathbf{A}_{1}^{2}, \\
\mathbf{A}_{1}=(\operatorname{grad} \mathbf{V})+(\operatorname{grad} \mathbf{V})^{\top}, \\
\mathbf{A}_{2}=\frac{d \mathbf{A}_{1}}{d t}+\mathbf{A}_{1}(\operatorname{grad} \mathbf{V})+(\operatorname{grad} \mathbf{V})^{\top} \mathbf{A}_{1},
\end{gathered}
$$


where $\mu,-p \mathbf{I}, \alpha_{j}(j=1,2), \mathbf{A}_{1}$, and $\mathbf{A}_{2}$ are, respectively, the dynamic viscosity, spherical stress, normal stress moduli, and first two Rivlin-Ericksen tensors. The thermodynamic analysis of model (2.6) has been discussed in detail by Dunn and Fosdick [4]. The Clausius-Duhem inequality and the assumption that the Helmholtz free energy is a minimum in equilbrium provide the following restrictions [5]:

$$
\mu \geq 0, \quad \alpha_{1} \geq 0, \quad \alpha_{1}+\alpha_{2}=0 .
$$

It is evident from (2.1) and (2.3) that

$$
\frac{\partial w^{\prime}}{\partial z^{\prime}}=0
$$

The above equation shows that $w^{\prime}$ is a function of time. Following Messiha [18] and Soundalgekar and Puri [23] we take

$$
w^{\prime}=-W_{0}^{\prime}\left(1+\epsilon A e^{i \omega^{\prime} t^{\prime}}\right)
$$

In above equation $W_{0}^{\prime}$ is nonzero constant mean suction velocity, $A$ is real positive constant, $\epsilon$ is small such that $\epsilon A \leq 1$, and negative sign indicates that suction velocity normal to the plate is directed towards the plate. From (2.1), (2.4), (2.6), (2.8), and (2.11) we get

$$
\begin{aligned}
& \frac{\partial u^{\prime}}{\partial t^{\prime}}-W_{0}^{\prime}\left(1+\epsilon A e^{i \omega^{\prime} t^{\prime}}\right) \frac{\partial u^{\prime}}{\partial z^{\prime}}-2 \Omega v^{\prime}=-\frac{1}{\rho^{\prime}} \frac{\partial \hat{p}}{\partial x^{\prime}}+v \frac{\partial^{2} u^{\prime}}{\partial z^{\prime 2}}+\alpha^{*} \frac{\partial^{3} u^{\prime}}{\partial z^{\prime 2} \partial t^{\prime}} \\
&-\alpha^{*} W_{0}^{\prime}\left(1+\epsilon A e^{i \omega^{\prime} t^{\prime}}\right) \frac{\partial^{3} u^{\prime}}{\partial z^{\prime 3}} \\
& \frac{\partial v^{\prime}}{\partial t^{\prime}}-W_{0}^{\prime}\left(1+\epsilon A e^{i \omega^{\prime} t^{\prime}}\right) \frac{\partial v^{\prime}}{\partial z^{\prime}} 2 \Omega u^{\prime}=-\frac{1}{\rho^{\prime}} \frac{\partial \hat{p}}{\partial y^{\prime}}+v \frac{\partial^{2} v^{\prime}}{\partial z^{\prime 2}}+\alpha^{*} \frac{\partial^{3} v^{\prime}}{\partial z^{\prime 2} \partial t^{\prime}} \\
&-\alpha^{*} W_{0}^{\prime}\left(1+\epsilon A e^{i \omega^{\prime} t^{\prime}}\right) \frac{\partial^{3} v^{\prime}}{\partial z^{\prime 3}}, \\
& \frac{\partial w^{\prime}}{\partial t^{\prime}}=-\frac{1}{\rho^{\prime}} \frac{\partial \hat{p}}{\partial z^{\prime}}
\end{aligned}
$$

subject to the boundary conditions

$$
\begin{gathered}
u^{\prime}=v^{\prime}=0 \quad \text { at } z^{\prime}=0, \\
u^{\prime} \longrightarrow U^{\prime}\left(t^{\prime}\right), \quad v^{\prime} \longrightarrow 0 \quad \text { as } z^{\prime} \longrightarrow \infty,
\end{gathered}
$$

where $U^{\prime}\left(t^{\prime}\right)$ is the free stream velocity and modified pressure

$$
\begin{gathered}
\hat{p}=p-\frac{1}{2} \rho^{\prime} \Omega^{2} r^{2}-\left(2 \alpha_{1}+\alpha_{2}\right)\left[\left(\frac{\partial u^{\prime}}{\partial z^{\prime}}\right)^{2}+\left(\frac{\partial v^{\prime}}{\partial z^{\prime}}\right)^{2}\right], \\
v=\frac{\mu}{\rho^{\prime}}, \quad \alpha^{*}=\frac{\alpha_{1}}{\rho^{\prime}} .
\end{gathered}
$$


558 Rotating second-grade flow with suction

In view of (2.11) and (2.14), $\partial \hat{p} / \partial z^{\prime}$ is small in the boundary and hence can be ignored $[8,18,23]$. The modified pressure $\hat{p}$ is assumed constant along any normal and is given by its value outside the boundary layer. Equations (2.12) and (2.13) for the free stream yields

$$
\begin{gathered}
-\frac{1}{\rho^{\prime}} \frac{\partial \hat{p}}{\partial x^{\prime}}=\frac{d U^{\prime}}{d t^{\prime}}, \\
-\frac{1}{\rho^{\prime}} \frac{\partial \hat{p}}{\partial y^{\prime}}=2 \Omega U^{\prime} .
\end{gathered}
$$

Making use of (2.18) and (2.19) into (2.12) and (2.13), we have

$$
\begin{aligned}
\frac{\partial u^{\prime}}{\partial t^{\prime}}-W_{0}^{\prime}\left(1+\epsilon A e^{i \omega^{\prime} t^{\prime}}\right) \frac{\partial u^{\prime}}{\partial z^{\prime}}-2 \Omega v^{\prime}= & \frac{d U^{\prime}}{d t^{\prime}}+v \frac{\partial^{2} u^{\prime}}{\partial z^{\prime 2}}+\alpha^{*} \frac{\partial^{3} u^{\prime}}{\partial z^{\prime 2} \partial t^{\prime}} \\
& -\alpha^{*} W_{0}^{\prime}\left(1+\epsilon A e^{i \omega^{\prime} t^{\prime}}\right) \frac{\partial^{3} u^{\prime}}{\partial z^{\prime 3}}, \\
\frac{\partial v^{\prime}}{\partial t^{\prime}}-W_{0}^{\prime}\left(1+\epsilon A e^{i \omega^{\prime} t^{\prime}}\right) \frac{\partial v^{\prime}}{\partial z^{\prime}} 2 \Omega u^{\prime}= & 2 \Omega U^{\prime}+v \frac{\partial^{2} v^{\prime}}{\partial z^{\prime 2}}+\alpha^{*} \frac{\partial^{3} v^{\prime}}{\partial z^{\prime 2} \partial t^{\prime}} \\
& -\alpha^{*} W_{0}^{\prime}\left(1+\epsilon A e^{i \omega^{\prime} t^{\prime}}\right) \frac{\partial^{3} v^{\prime}}{\partial z^{\prime 3}},
\end{aligned}
$$

where $U^{\prime}$ is periodic free stream velocity given by

$$
U^{\prime}\left(t^{\prime}\right)=U_{0}^{\prime}\left(1+\epsilon e^{i \omega^{\prime} t^{\prime}}\right),
$$

where $U_{0}^{\prime}$ is the reference velocity.

With the help of (2.22), (2.20), (2.21), and boundary conditions (2.15) become

$$
\begin{gathered}
\frac{\partial F^{\prime}}{\partial t^{\prime}}-W_{0}^{\prime}\left(1+\epsilon A e^{i \omega^{\prime} t^{\prime}}\right) \frac{\partial F^{\prime}}{\partial z^{\prime}}+2 i \Omega F^{\prime} \\
=U_{0}^{\prime} i \omega^{\prime} \epsilon e^{i l \omega^{\prime} t^{\prime}}+v \frac{\partial^{2} F^{\prime}}{\partial z^{\prime 2}}+2 i \Omega U_{0}^{\prime}\left(1+\epsilon e^{i \omega^{\prime} t^{\prime}}\right)+\alpha^{*} \frac{\partial^{3} F^{\prime}}{\partial z^{\prime 2} \partial t^{\prime}} \\
-\alpha^{*} W_{0}^{\prime}\left(1+\epsilon A e^{i \omega^{\prime} t^{\prime}}\right) \frac{\partial^{3} F^{\prime}}{\partial z^{\prime 3}} \\
F^{\prime}=0 \quad \text { at } z^{\prime}=0, \\
F^{\prime}=U_{o}^{\prime}\left(1+\epsilon e^{i \omega^{\prime} t^{\prime}}\right) \quad \text { as } z^{\prime} \longrightarrow \infty,
\end{gathered}
$$

where

$$
F^{\prime}=u^{\prime}+i v^{\prime}
$$

Introducing the nondimensional variables

$$
\begin{gathered}
\eta=\frac{z^{\prime} W_{o}^{\prime}}{v}, \quad t=\frac{W_{o}^{\prime 2} t^{\prime}}{4 v}, \quad \omega=\frac{4 v \omega^{\prime}}{W_{o}^{\prime 2}}, \quad U=\frac{U^{\prime}}{U_{o}^{\prime}}, \\
u=\frac{u^{\prime}}{U_{o}^{\prime}}, \quad v=\frac{v^{\prime}}{U_{o}^{\prime}}, \quad F=\frac{F^{\prime}}{U_{o}^{\prime}},
\end{gathered}
$$


the boundary value problem consisting of (2.23) and conditions (2.24) yields

$$
\begin{aligned}
& \frac{1}{4} \frac{\partial F}{\partial t}-\left(1+\epsilon A e^{i \omega t}\right) \frac{\partial F}{\partial \eta}+2 i N F=\frac{1}{4}\left(i \omega \epsilon e^{i \omega t}\right)+2 i N\left(1+\epsilon e^{i \omega t}\right)+\frac{\partial^{2} F}{\partial \eta^{2}} \\
& +\alpha\left(\frac{1}{4} \frac{\partial^{3} F}{\partial \eta^{2} \partial t}-\left(1+\epsilon A e^{i \omega t}\right) \frac{\partial^{3} F}{\partial \eta^{3}}\right), \\
& F=0 \quad \text { at } \eta=0 \text {, } \\
& F \longrightarrow 1+\epsilon e^{i \omega t} \text { as } \eta \longrightarrow \infty,
\end{aligned}
$$

where

$$
\alpha=\frac{\alpha^{*} W_{o}^{\prime 2}}{v^{2}}, \quad N=\frac{\Omega \nu}{W_{o}^{\prime 2}} .
$$

\section{Analytical solution}

The solution of (2.27) subject to conditions (2.28) is written as

$$
F(\eta, t)=f_{1}(\eta)+\epsilon e^{i \omega t} f_{2}(\eta)
$$

Using above equation into (2.27) and separating the harmonic and nonharmonic terms we obtain

$$
\begin{gathered}
\alpha \frac{d^{3} f_{1}}{d \eta^{3}}-\frac{d^{2} f_{1}}{d \eta^{2}}-\frac{d f_{1}}{d \eta}+i N f_{1}=i N, \\
\alpha \frac{d^{3} f_{2}}{d \eta^{3}}-\left(1+\frac{i \alpha \omega}{4}\right) \frac{d^{2} f_{2}}{d \eta^{2}}-\frac{d f_{2}}{d \eta}+i N_{1} f_{2}=i N_{1}+A \frac{d f_{1}}{d \eta}-\alpha A \frac{d^{3} f_{1}}{d \eta^{3}},
\end{gathered}
$$

where

$$
N_{1}=N+\frac{\omega}{4}
$$

The corresponding boundary conditions are

$$
\begin{gathered}
f_{1}=0 \quad \text { at } \eta=0, \\
f_{1} \longrightarrow 1 \quad \text { as } \eta \longrightarrow \infty, \\
f_{2}=0 \quad \text { at } \eta=0, \\
f_{2} \longrightarrow 1 \text { as } \eta \longrightarrow \infty .
\end{gathered}
$$


It is worth emphasizing that (3.2) for second-grade fluid are third order (one order higher than the Navier-Stokes equation). Thus, one needs three conditions for the unique solution whereas two conditions are prescribed. One possible way to overcome this difficulty is to employ a perturbation analysis (as in Beard and Walters [2], Soundalgekar and Puri [23], Kaloni [14], and Hayat et al. [8]) and take the solution as follows

$$
\begin{aligned}
& f_{1}=f_{01}+\alpha f_{11}+o\left(\alpha^{2}\right), \\
& f_{2}=f_{02}+\alpha f_{12}+o\left(\alpha^{2}\right) .
\end{aligned}
$$

Substituting (3.5) into (3.2), (3.4), equating the coefficients of $\alpha$, and then solving the corresponding problems we have for $f_{1}$ and $f_{2}$

$$
\begin{gathered}
f_{1}=1-(1+\alpha \eta L) e^{-h \eta}, \\
f_{2}=1-S e^{-g \eta}-(1-S) e^{-h \eta}+\alpha\left[c_{5} e^{-g \eta}-\eta M e^{-g \eta}-\left(\eta c_{3}+c_{5}\right) e^{-h \eta}\right],
\end{gathered}
$$

and so from (3.1),

$$
F=1-(1+\alpha \eta L) e^{-h \eta}+\epsilon e^{i \omega t}\left[\begin{array}{c}
1-S e^{-g \eta}-(1-S) e^{-h \eta} \\
+\alpha\left\{c_{5} e^{-g \eta}-\eta M e^{-g \eta}-\left(\eta c_{3+} c_{5}\right) e^{-h \eta}\right\}
\end{array}\right],
$$

which upon separating real and imaginary parts gives

$$
\begin{aligned}
& u=u_{0}+\epsilon e^{i \omega t} u_{1}=1-e^{-h_{r} \eta}\left(\left(1+\alpha \eta L_{r}\right) \cosh _{i} \eta+\alpha \eta L_{i} \sinh _{i} \eta\right) \\
& +\epsilon e^{i \omega t}\left[\begin{array}{c}
1-e^{-g_{r} \eta}\left(\left(1-\frac{4 A h_{i}}{\omega}\right) \cos g_{i} \eta+\frac{4 A h_{r}}{\omega} \sin g_{i} \eta\right) \\
+e^{-h_{r} \eta}\left(\frac{4 A h_{i}}{\omega} \cosh _{i} \eta-\frac{4 A h_{r}}{\omega} \sinh _{i} \eta\right) \\
+\alpha e^{-g_{r} \eta}\left(c_{5 r} \cos g_{i} \eta+c_{5 i} \sin g_{i} \eta\right) \\
-\alpha \eta e^{-g_{r} \eta}\left(M_{r} \cos g_{i} \eta+M_{i} \sin g_{i} \eta\right) \\
-\alpha e^{-h_{r} \eta}\left(\left(\eta c_{3 r}+c_{5 r}\right) \cos h_{i} \eta+\left(\eta c_{3 i}+c_{5 i}\right) \sin h_{i} \eta\right)
\end{array}\right],
\end{aligned}
$$


T. Hayat et al. 561

$$
\begin{aligned}
& v=v_{0}+\epsilon e^{i \omega t} v_{1}=e^{-h_{r} \eta}\left(\left(1+\alpha \eta L_{r}\right) \sinh _{i} \eta+\alpha \eta L_{i} \cosh _{i} \eta\right) \\
& +\epsilon e^{i \omega t}\left[\begin{array}{c}
e^{-g_{r} \eta}\left(\left(1-\frac{4 A h_{i}}{\omega}\right) \sin g_{i} \eta-\frac{4 A h_{r}}{\omega} \cos g_{i} \eta\right) \\
-e^{-h_{r} \eta}\left(\frac{4 A h_{i}}{\omega} \sinh _{i} \eta+\frac{4 A h_{r}}{\omega} \cosh _{i} \eta\right) \\
+\alpha e^{-g_{r} \eta}\left(c_{5 i} \cos g_{i} \eta-c_{5 r} \sin g_{i} \eta\right) \\
-\alpha \eta e^{-g_{r} \eta}\left(M_{i} \cos g_{i} \eta-M_{r} \sin g_{i} \eta\right) \\
-\alpha e^{-h_{r} \eta}\left(\left(\eta c_{3 i}+c_{5 i}\right) \cos h_{i} \eta-\left(\eta c_{3 r}+c_{5 r}\right) \sin h_{i} \eta\right)
\end{array}\right],
\end{aligned}
$$

where

$$
\begin{aligned}
& h=h_{r}+i h_{i}=\frac{1+\sqrt{1+4 i N}}{2}, \\
& h_{r}=\frac{1}{2}+\frac{1}{2}\left[\frac{1}{2}\left(1+\sqrt{1+16 N^{2}}\right)\right]^{2}, \quad h_{i}=\frac{1}{2}\left[\frac{1}{2}\left(-1+\sqrt{1+16 N^{2}}\right)\right]^{2}, \\
& a=\left[\frac{1}{2}\left(1+\sqrt{1+16 N^{2}}\right)\right]^{2}, \quad b=\left[\frac{1}{2}\left(-1+\sqrt{1+16 N^{2}}\right)\right]^{2}, \\
& r=a^{2}+b^{2}=\sqrt{1+16 N^{2}}, \quad g=g_{r}+i g_{i}=\frac{1+\sqrt{1+4 i N_{1}}}{2}, \\
& g_{r}=\frac{1}{2}+\frac{1}{2}\left[\frac{1}{2}\left(1+\sqrt{1+16 N_{1}^{2}}\right)\right]^{2}, \quad g_{i}=\frac{1}{2}\left[\frac{1}{2}\left(-1+\sqrt{1+16 N_{1}^{2}}\right)\right]^{2}, \\
& a_{1}=\left[\frac{1}{2}\left(1+\sqrt{1+16 N_{1}^{2}}\right)\right]^{2}, \quad b_{1}=\left[\frac{1}{2}\left(-1+\sqrt{1+16 N_{1}^{2}}\right)\right]^{2}, \\
& r_{1}=a_{1}^{2}+b_{1}^{2}=\sqrt{1+16 N_{1}^{2}}, \quad S=S_{r}+i S_{i}=1-\frac{4 i A h}{\omega}, \\
& S_{r}=1+\frac{4 A h_{i}}{\omega}, \quad S_{i}=\frac{4 A h_{r}}{\omega}, \quad L=L_{r}+i L_{i}=\frac{h^{3}}{\sqrt{1+4 i N}}, \\
& L_{r}=\frac{1}{r}\left[\begin{array}{c}
\frac{1}{4}\left(\frac{r+1}{2}\right)^{1 / 2}\left(1+\left(\frac{r+1}{2}\right)\right)+\frac{1}{2}-2 N^{2} \\
-\frac{7}{4}\left(\frac{r-1}{2}\right)\left(\frac{r+1}{2}\right)^{1 / 2}
\end{array}\right], \\
& L_{i}=\frac{1}{r}\left[\begin{array}{c}
\frac{1}{4}\left(\frac{r-1}{2}\right)^{1 / 2}\left(1-\left(\frac{r-1}{2}\right)\right)+\frac{5 N}{2} \\
+\frac{3}{2}\left(\frac{r+1}{2}\right)\left(\frac{r-1}{2}\right)^{1 / 2}
\end{array}\right], \\
& M=M_{r}+i M_{i}=\frac{g^{2}(g+i \omega / 4)(1-4 i A h / \omega)}{\sqrt{1+4 i N_{1}}},
\end{aligned}
$$




$$
\begin{aligned}
& M_{r}=\frac{1}{r_{1}}\left[\begin{array}{c}
\frac{1}{4}\left(\frac{r_{1}+1}{2}\right)^{1 / 2}+\frac{1}{8}\left(r_{1}+1\right)\left(\frac{r_{1}+1}{2}\right)^{1 / 2}+\frac{r_{1}}{2} \\
+\frac{3}{8}\left(r_{1}-1\right)\left(\frac{r_{1}+1}{2}\right)^{1 / 2}-N_{1}^{2}\left(\frac{r_{1}+1}{2}\right)^{1 / 2} \\
+\frac{A h_{i}}{\omega}\left(\begin{array}{c}
\left(\frac{r_{1}+1}{2}\right)^{1 / 2}+\left(\frac{r_{1}+1}{2}\right)\left(\frac{r_{1}+1}{2}\right)^{1 / 2} \\
+2 r_{1}+\frac{3}{2}\left(r_{1}-1\right)\left(\frac{r_{1}+1}{2}\right)^{1 / 2} \\
-4 N_{1}^{2}\left(\frac{r_{1}+1}{2}\right)^{1 / 2}
\end{array}\right. \\
+\frac{A h r}{\omega}\left(\begin{array}{c}
2 N_{1}\left(r_{1}+1\right)+\frac{3}{2}\left(r_{1}+1\right)\left(\frac{r_{1}-1}{2}\right)^{1 / 2} \\
-\left(\frac{r_{1}-1}{2}\right)^{1 / 2}+\left(\frac{r_{1}-1}{2}\right)^{\left(\frac{r_{1}-1}{2}\right)^{1 / 2}}
\end{array}\right.
\end{array}\right], \\
& M_{i}=\frac{1}{r_{1}}\left[\begin{array}{c}
2 N_{1} r_{1}-\frac{1}{4}\left(\frac{r_{1}-1}{2}\right)^{1 / 2}+\frac{3}{8}\left(r_{1}+1\right)\left(\frac{r_{1}+1}{2}\right)^{1 / 2} \\
+\frac{3}{8}\left(r_{1}-1\right)\left(\frac{r_{1}-1}{2}\right)^{1 / 2}+N_{1}^{2}\left(\frac{r_{1}-1}{2}\right)^{1 / 2} \\
+\frac{A h_{i}}{8 N_{1}+4 N_{1} r_{1}+\left(\frac{r_{1}-1}{2}\right)^{1 / 2}}+\left(\begin{array}{c}
\left.\frac{r_{1}-1}{2}\right)\left(\frac{r_{1}-1}{2}\right)^{1 / 2}+4 N_{1}^{2}\left(\frac{r_{1}-1}{2}\right)^{1 / 2} \\
+\frac{5}{2}\left(r_{1}+1\right)\left(\frac{r_{1}-1}{2}\right)^{1 / 2} \\
-3 N_{1}\left(r_{1}-1\right)-2 r_{1}
\end{array}\right. \\
+\frac{A h r}{\omega}\left(\begin{array}{c}
-\left(\frac{r_{1}+1}{2}\right)^{1 / 2}\left(1+\left(\frac{r_{1}+1}{2}\right)\right) \\
+4 N_{1}^{2}\left(\frac{r_{1}+1}{2}\right)^{1 / 2}
\end{array}\right.
\end{array}\right], \\
& c_{5}=c_{5 r}+i c_{5 i}=\left(c_{1 r}+c_{2 r}+c_{4 r}\right)+i\left(c_{1 i}+c_{2 i}+c_{4 i}\right) \text {, } \\
& c_{1}=c_{1 r}+i c_{1 i}=\frac{4 h^{3}}{\iota \omega}(A-(1-S)) \text {, } \\
& c_{1 r}=4\left(h_{i}^{3}-3 h_{r}^{2} h_{i}\right)\left(A+\frac{4 A h_{i}}{\omega}\right)-16 \frac{A h_{r}}{\omega}\left(h_{r}^{3}-3 h_{i}^{2} h_{r}\right) \text {, } \\
& c_{1 i}=-4\left(h_{r}^{3}-3 h_{i}^{2} h_{r}\right)\left(A+\frac{4 A h_{i}}{\omega}\right)-16 \frac{A h_{r}}{\omega}\left(h_{r}^{3}-3 h_{r}^{2} h_{i}\right) \text {, } \\
& c_{2}=c_{2 r}+i c_{2 i}=\frac{4 A\left(h^{3}+L\right)}{i \omega}, \\
& c_{2 r}=\frac{4 A}{\omega}\left(L_{i}\left(h_{r}^{3}-3 h_{i}^{2} h_{r}\right)-L_{r}\left(h_{i}^{3}-3 h_{r}^{2} h_{i}\right)\right),
\end{aligned}
$$


T. Hayat et al. 563

$$
\begin{gathered}
c_{2 i}=-\frac{4 A}{\omega}\left(L_{r}\left(h_{r}^{3}-3 h_{i}^{2} h_{r}\right)+L_{i}\left(h_{i}^{3}-3 h_{r}^{2} h_{i}\right)\right), \\
c_{3}=c_{3 r}+i c_{3 i}=\frac{4 A h L}{i \omega}, \\
c_{3 r}=\frac{4 A}{\omega}\left(h_{i} L_{r}+h_{r} L_{i}\right), \quad c_{3 i}=-\frac{4 A}{\omega}\left(h_{r} L_{r}-h_{i} L_{i}\right), \\
c_{4}=c_{4 r}+i c_{4 i}=\frac{16 A h L(1-2 h)}{\omega^{2}}, \\
c_{4 r}=\frac{16 A}{\omega^{2}}\left(\left(1-2 h_{r}\right)\left(h_{r} L_{r}-h_{i} L_{i}\right)+2 h_{i}\left(h_{r} L_{i}+h_{i} L_{r}\right)\right), \\
c_{4 i}=\frac{16 A}{\omega^{2}}\left(\left(1-2 h_{r}\right)\left(h_{r} L_{i}+h_{i} L_{r}\right)-2 h_{i}\left(h_{r} L_{r}-h_{i} L_{i}\right)\right) .
\end{gathered}
$$

The drag $P_{x z}$ and lateral stress $P_{y z}$ at the plate in nondimensional form can be written, respectively, as

$$
\begin{aligned}
& P_{x z}=\frac{P_{x^{\prime} z^{\prime}}^{\prime}}{U_{0}^{\prime} W_{0}^{\prime} \rho^{\prime}}=\frac{\partial u}{\partial \eta}-\frac{\alpha}{4}\left[\frac{\partial^{2} u}{\partial \eta \partial t}-4\left(1+\epsilon A e^{i \omega t}\right) \frac{\partial^{2} u}{\partial \eta^{2}}\right] \\
& P_{y z}=\frac{P_{y^{\prime} z^{\prime}}^{\prime}}{U_{0}^{\prime} W_{0}^{\prime} \rho^{\prime}}=\frac{\partial v}{\partial \eta}-\frac{\alpha}{4}\left[\frac{\partial^{2} v}{\partial \eta \partial t}-4\left(1+\epsilon A e^{i \omega t}\right) \frac{\partial^{2} v}{\partial \eta^{2}}\right] .
\end{aligned}
$$

The above equations after using (3.8) and (3.9) give

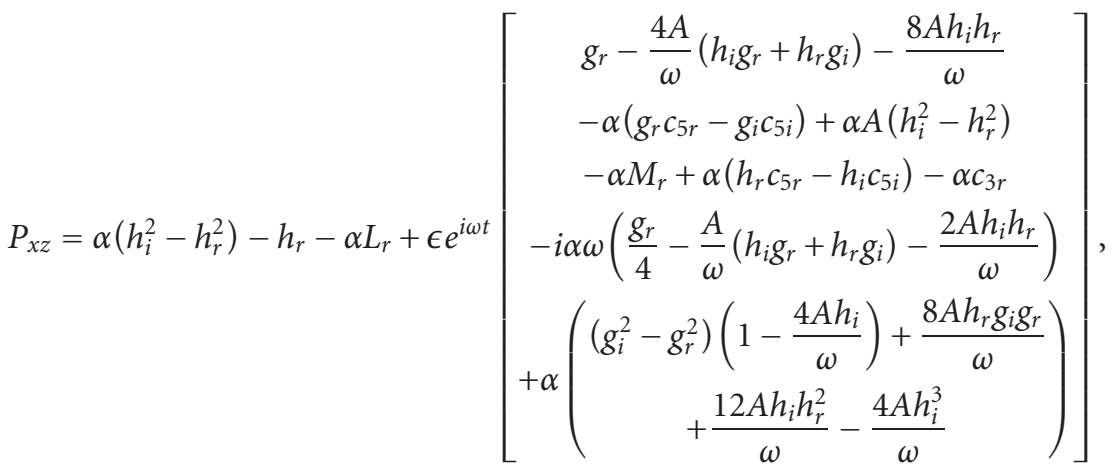

$$
\begin{aligned}
& P_{y z}=h_{i}-\alpha L_{i}-2 \alpha h_{i} h_{r}+\epsilon e^{i \omega t}\left[\begin{array}{c}
g_{i}+\frac{4 A}{\omega}\left(h_{r} g_{r}-h_{i} g_{i}\right)+\frac{4 A}{\omega}\left(h_{r}^{2}-h_{i}^{2}\right) \\
-\alpha\left(g_{r} c_{5 i}+g_{i} c_{5 r}\right)-2 \alpha A h_{i} h_{r} \\
-\alpha M_{i}+\alpha\left(h_{r} c_{5 i}+h_{i} c_{5 r}\right)-\alpha c_{3 i} \\
-i \alpha \omega\left(g_{i}+\frac{4 A}{\omega}\left(h_{r} g_{r}-h_{i} g_{i}\right)+\frac{4 A}{\omega}\left(h_{r}^{2}-h_{i}^{2}\right)\right) \\
+\alpha\left(\begin{array}{c}
-2 g_{i} g_{r}+\frac{8 A h_{i} g_{i} g_{r}}{\omega}-\frac{4 A h_{r}}{\omega}\left(g_{r}^{2}-g_{i}^{2}\right) \\
+\frac{12 A h_{r} h_{i}^{2}}{\omega}-\frac{4 A h_{r}^{3}}{\omega}
\end{array}\right)
\end{array}\right] \text {. }
\end{aligned}
$$


564 Rotating second-grade flow with suction

The above equations can also be written as

$$
\begin{gathered}
P_{x z}=\alpha\left(h_{i}^{2}-h_{r}^{2}\right)-h_{r}-\alpha L_{r}+\epsilon|B| \cos (\omega t+\beta), \\
P_{y z}=h_{i}-\alpha L_{i}-2 \alpha h_{i} h_{r}+\epsilon\left|B_{1}\right| \cos (\omega t+\gamma),
\end{gathered}
$$

where

$$
\begin{aligned}
& B=B_{r}+i B_{i}=\left[\begin{array}{c}
g_{r}-\frac{4 A}{\omega}\left(h_{i} g_{r}+h_{r} g_{i}\right)-\frac{8 A h_{i} h_{r}}{\omega} \\
-\alpha\left(g_{r} c_{5 r}-g_{i} c_{5 i}\right)+\alpha A\left(h_{i}^{2}-h_{r}^{2}\right) \\
-\alpha M_{r}+\alpha\left(h_{r} c_{5 r}-h_{i} c_{5 i}\right)-\alpha c_{3 r} \\
-i \alpha \omega\left(\frac{g_{r}}{4}-\frac{A}{\omega}\left(h_{i} g_{r}+h_{r} g_{i}\right)-\frac{2 A h_{i} h_{r}}{\omega}\right) \\
+\alpha\left(\begin{array}{c}
\left(g_{i}^{2}-g_{r}^{2}\right)\left(1-\frac{4 A h_{i}}{\omega}\right)+\frac{8 A h_{r} g_{i} g_{r}}{\omega} \\
+\frac{12 A h_{i} h_{r}^{2}}{\omega}-\frac{4 A h_{i}^{3}}{\omega}
\end{array}\right)
\end{array}\right], \\
& B_{r}=\left[\begin{array}{c}
g_{r}-\frac{4 A}{\omega}\left(h_{i} g_{r}+h_{r} g_{i}\right)-\frac{8 A h_{i} h_{r}}{\omega} \\
-\alpha\left(g_{r} c_{5 r}-g_{i} c_{5 i}\right)+\alpha A\left(h_{i}^{2}-h_{r}^{2}\right) \\
-\alpha M_{r}+\alpha\left(h_{r} c_{5 r}-h_{i} c_{5 i}\right)-\alpha c_{3 r} \\
+\alpha\left(\begin{array}{c}
\left(g_{i}^{2}-g_{r}^{2}\right)\left(1-\frac{4 A h_{i}}{\omega}\right)+\frac{8 A h_{r} g_{i} g_{r}}{\omega} \\
+\frac{12 A h_{i} h_{r}^{2}}{\omega}-\frac{4 A h_{i}^{3}}{\omega}
\end{array}\right)
\end{array}\right], \\
& B_{i}=\alpha \omega\left(\frac{g_{r}}{4}-\frac{A}{\omega}\left(h_{i} g_{r}+h_{r} g_{i}\right)-\frac{2 A h_{i} h_{r}}{\omega}\right), \\
& B_{1}=B_{1 r}+i B_{1 i}=\left[\begin{array}{c}
g_{i}+\frac{4 A}{\omega}\left(h_{r} g_{r}-h_{i} g_{i}\right)+\frac{4 A}{\omega}\left(h_{r}^{2}-h_{i}^{2}\right) \\
-\alpha\left(g_{r} c_{5 i}+g_{i} c_{5 r}\right)-2 \alpha A h_{i} h_{r} \\
-\alpha M_{i}+\alpha\left(h_{r} c_{5 i}+h_{i} c_{5 r}\right)-\alpha c_{3 i} \\
-i \alpha \omega\left(g_{i}+\frac{4 A}{\omega}\left(h_{r} g_{r}-h_{i} g_{i}\right)+\frac{4 A}{\omega}\left(h_{r}^{2}-h_{i}^{2}\right)\right) \\
+\alpha\left(\begin{array}{c}
-2 g_{i} g_{r}+\frac{8 A h_{i} g_{i} g_{r}}{\omega}-\frac{4 A h_{r}}{\omega}\left(g_{r}^{2}-g_{i}^{2}\right) \\
+\frac{12 A h_{r} h_{i}^{2}}{\omega}-\frac{4 A h_{r}^{3}}{\omega}
\end{array}\right)
\end{array}\right] \text {, } \\
& \beta=\tan ^{-1}\left(\frac{B_{i}}{B_{r}}\right), \quad \gamma=\tan ^{-1}\left(\frac{B_{1 i}}{B_{1 r}}\right),
\end{aligned}
$$




$$
B_{1 r}=\left[\begin{array}{c}
g_{i}+\frac{4 A}{\omega}\left(h_{r} g_{r}-h_{i} g_{i}\right)+\frac{4 A}{\omega}\left(h_{r}^{2}-h_{i}^{2}\right) \\
-\alpha\left(g_{r} c_{5 i}+g_{i} c_{5 r}\right)-2 \alpha A h_{i} h_{r} \\
-\alpha M_{i}+\alpha\left(h_{r} c_{5 i}+h_{i} c_{5 r}\right)-\alpha c_{3 i} \\
+\alpha\left(\begin{array}{c}
-2 g_{i} g_{r}+\frac{8 A h_{i} g_{i} g_{r}}{\omega}-\frac{4 A h_{r}}{\omega}\left(g_{r}^{2}-g_{i}^{2}\right) \\
+\frac{12 A h_{r} h_{i}^{2}}{\omega}-\frac{4 A h_{r}^{3}}{\omega}
\end{array}\right)
\end{array}\right],
$$

We now proceed to derive the energy equation appropriate for the problem under consideration. We start with the energy equation (2.5). It follows from (2.5), (2.6), (2.7), (2.8), and (2.9) and $\mathbf{L}=\operatorname{grad} \mathbf{V}$ that

$$
\mathbf{T} \cdot \mathbf{L}=\mu\left[\left(\frac{\partial u^{\prime}}{\partial z^{\prime}}\right)^{2}+\left(\frac{\partial v^{\prime}}{\partial z^{\prime}}\right)^{2}\right]+\alpha\left[\begin{array}{c}
\frac{\partial u^{\prime}}{\partial z^{\prime}}\left(\frac{\partial^{2} u^{\prime}}{\partial t^{\prime} \partial z^{\prime}}+w^{\prime} \frac{\partial^{2} u^{\prime}}{\partial z^{\prime 2}}\right) \\
+\frac{\partial v^{\prime}}{\partial z^{\prime}}\left(\frac{\partial^{2} v^{\prime}}{\partial t^{\prime} \partial z^{\prime}}+w^{\prime} \frac{\partial^{2} v^{\prime}}{\partial z^{\prime 2}}\right)
\end{array}\right]
$$

Following the thermodynamical considerations given in Dunn and Fosdick [4] for fluids of second grade and representing $\mathbf{q}$ by Fourier's law with a constant thermal conductivity, $k,(2.5)$ reduces to

$$
\rho^{\prime} c\left[\frac{\partial T}{\partial t^{\prime}}+w^{\prime} \frac{\partial T}{\partial z^{\prime}}\right]-k \frac{\partial^{2} T}{\partial z^{\prime 2}}=\mu\left[\left(\frac{\partial u^{\prime}}{\partial z^{\prime}}\right)^{2}+\left(\frac{\partial v^{\prime}}{\partial z^{\prime}}\right)^{2}\right]+\alpha_{1}\left[\begin{array}{c}
\frac{\partial u^{\prime}}{\partial z^{\prime}}\left(\frac{\partial^{2} u^{\prime}}{\partial t^{\prime} \partial z^{\prime}}+w^{\prime} \frac{\partial^{2} u^{\prime}}{\partial z^{\prime 2}}\right) \\
+\frac{\partial v^{\prime}}{\partial z^{\prime}}\left(\frac{\partial^{2} v^{\prime}}{\partial t^{\prime} \partial z^{\prime}}+w^{\prime} \frac{\partial^{2} v^{\prime}}{\partial z^{\prime 2}}\right)
\end{array}\right]
$$

where $c$ is the specific heat. The boundary conditions for the temperature are

$$
\begin{gathered}
T=T_{0} \quad \text { at } z^{\prime}=0, \\
T \longrightarrow T_{\infty} \quad \text { as } z^{\prime} \longrightarrow \infty .
\end{gathered}
$$

Using

$$
\theta=\frac{T-T_{0}}{T_{\infty}-T_{0}},
$$


(3.17) and boundary conditions (3.18) become

$$
\begin{gathered}
-\frac{\partial^{2} \theta}{\partial \eta^{2}}-P_{r}\left(1+\epsilon A e^{i \omega t}\right) \frac{\partial \theta}{\partial \eta}+\frac{P_{r}}{4} \frac{\partial \theta}{\partial t}=E_{c}\left[\left(\frac{\partial u}{\partial \eta}\right)^{2}+\left(\frac{\partial v}{\partial \eta}\right)^{2}\right]+P\left[\begin{array}{c}
\frac{\partial u}{\partial \eta} \frac{\partial^{2} u}{\partial \eta \partial t}+\frac{\partial v}{\partial \eta} \frac{\partial^{2} v}{\partial \eta \partial t} \\
-\left(1+\epsilon A e^{i \omega t}\right) \frac{\partial u}{\partial \eta} \frac{\partial^{2} u}{\partial \eta^{2}} \\
-\left(1+\epsilon A e^{i \omega t}\right) \frac{\partial v}{\partial \eta} \frac{\partial^{2} v}{\partial \eta^{2}}
\end{array}\right], \\
\theta=0 \text { at } \eta=0, \\
\theta \longrightarrow 1 \text { at } \eta \longrightarrow \infty,
\end{gathered}
$$

in which

$$
\begin{gathered}
P_{r}=\frac{\mu c}{k}, \quad E_{c}=\frac{k^{*} U_{0}^{2}}{\left(T_{\infty}-T_{0}\right)}, \\
P=\frac{\alpha U_{0}^{\prime 2} \mu}{k\left(T_{\infty}-T_{0}\right)} .
\end{gathered}
$$

We further assume that

$$
\theta=\theta_{0}+\epsilon e^{i \omega t} \theta_{1}
$$

Substituting (3.24) into (3.20) and boundary conditions (3.21), and equating the coefficients of the harmonic and nonharmonic term after neglecting the coefficients of $\epsilon^{2}$, we get

$$
\begin{gathered}
\frac{d^{2} \theta_{0}}{d \eta^{2}}+P_{r} \frac{d \theta_{0}}{d \eta}=-E_{c}\left[\left(\frac{d u_{1}}{d \eta}\right)^{2}+\left(\frac{d v_{1}}{d \eta}\right)^{2}\right]+P\left[\frac{d u_{1}}{d \eta} \frac{d^{2} u_{1}}{d \eta^{2}}+\frac{d v_{1}}{d \eta} \frac{d^{2} v_{1}}{d \eta^{2}}\right], \\
\frac{d^{2} \theta_{1}}{d \eta^{2}}+P_{r} \frac{d \theta_{1}}{d \eta}-\frac{P_{r}}{4} i \omega \theta_{1}=-P_{r} A \frac{d \theta_{0}}{d \eta}-2 E_{c}\left[\frac{d u_{1}}{d \eta} \frac{d u_{2}}{d \eta}+\frac{d v_{1}}{d \eta} \frac{d v_{2}}{d \eta}\right] \\
{\left[\begin{array}{c}
i \omega\left(\frac{d u_{1}}{d \eta} \frac{d u_{2}}{d \eta}+\frac{d v_{1}}{d \eta} \frac{d v_{2}}{d \eta}\right) \\
-\left(\frac{d u_{1}}{d \eta} \frac{d^{2} u_{2}}{d \eta^{2}}+\frac{d v_{1}}{d \eta} \frac{d^{2} v_{2}}{d \eta^{2}}\right) \\
-P\left(\frac{d u_{1}}{d \eta} \frac{d^{2} u_{1}}{d \eta^{2}}+\frac{d v_{1}}{d \eta} \frac{d^{2} v_{1}}{d \eta^{2}}\right) \\
-A \\
-\left(\frac{d u_{2}}{d \eta} \frac{d^{2} u_{1}}{d \eta^{2}}+\frac{d v_{2}}{d \eta} \frac{d^{2} v_{1}}{d \eta^{2}}\right)
\end{array}\right],} \\
\theta_{0}=0 \quad \text { at } \eta=0, \\
\theta_{0} \longrightarrow 1 \quad \text { at } \eta \longrightarrow \infty, \\
\theta_{1}=0 \quad \text { at } \eta=0, \\
\theta_{1} \longrightarrow 0 \quad \text { at } \eta \longrightarrow \infty .
\end{gathered}
$$


Solving (3.25) and (3.26) along with the boundary conditions (3.27), we obtain

$$
\begin{aligned}
& \theta_{0}=1-\left(1+d_{7}\right) e^{-P_{r} \eta}+\left(d_{7}+d_{8} \eta\right) e^{-2 h_{r} \eta}, \\
\theta_{1}= & -m_{16} e^{-f \eta}+\left(m_{7}+m_{9}+m_{8} \eta\right) e^{-2 h_{r} \eta} \\
+ & \left(m_{10}+m_{14}+m_{12} \eta\right) e^{-\left(h_{r}+g_{r}\right) \eta} \cos \left(h_{i}-g_{i}\right) \eta \\
+ & \left(m_{11}+m_{15}+m_{13} \eta\right) e^{-\left(h_{r}+g_{r}\right) \eta} \sin \left(h_{i}-g_{i}\right) \eta,
\end{aligned}
$$

where

$$
\begin{aligned}
& d_{1}=-E_{c}\left(h_{r}^{2}+h_{i}^{2}-2 \alpha L_{i} h_{i}\right), \quad d_{2}=-2 E_{c} \alpha L_{r}\left(h_{r}^{2}+h_{i}^{2}\right), \\
& d_{3}=P\left(-h_{r}^{3}+3 \alpha L_{r} h_{r}^{2}-h_{r} h_{i}^{2}+2 \alpha L_{i} h_{i} h_{r}+\alpha L_{r} h_{i}^{2}\right), \\
& d_{4}=-2 P \alpha L_{r} h_{r}\left(h_{r}^{2}+h_{i}^{2}\right), \quad d_{5}=d_{1}+d_{3}, \quad d_{6}=d_{2}+d_{4}, \\
& d_{7}=\frac{d_{5}}{4 h_{r}^{2}-2 P_{r} h_{r}}-\frac{d_{6}\left(4 h_{r}-P_{r}\right)}{\left(4 h_{r}^{2}-2 P_{r} h_{r}\right)^{2}}, \quad d_{8}=\frac{d_{6}}{\left(4 h_{r}^{2}-2 P_{r} h_{r}\right)}, \\
& d_{9}=\left[\begin{array}{c}
-\frac{4 A h_{r}}{\omega}\left(h_{r} g_{r}+h_{i}^{2}\right)+\left(1-\frac{4 A h_{i}}{\omega}\right)\left(h_{i} g_{r}-h_{r} g_{i}\right) \\
+\alpha\left(h_{r}\left(g_{r} c_{5 i}+g_{i} c_{5 r}\right)-h_{i}\left(g_{r} c_{5 r}-g_{i} c_{5 i}\right)\right) \\
+\alpha\left(h_{r} M_{i}-h_{i} M_{r}\right)+\frac{4 \alpha A h_{r}}{\omega}\left(g_{r} L_{r}-g_{i} L_{i}\right) \\
+\alpha\left(1-\frac{4 A h_{i}}{\omega}\right)\left(g_{i} L_{r}-g_{r} L_{i}\right)
\end{array}\right], \\
& d_{10}=\left[\begin{array}{c}
-\frac{4 \alpha A h_{r}^{2}}{\omega}\left(L_{r} g_{r}+L_{i} g_{i}\right)-\alpha h_{r}\left(1-\frac{4 A h_{i}}{\omega}\right)\left(L_{r} g_{i}-L_{i} g_{r}\right) \\
-\alpha\left(h_{r}\left(g_{r} M_{i}+g_{i} M_{r}\right)-h_{i}\left(g_{r} M_{r}-g_{i} M_{i}\right)\right) \\
-\frac{4 \alpha A h_{r} h_{i}}{\omega}\left(g_{i} L_{r}-g_{r} L_{i}\right)+\alpha h_{i}\left(1-\frac{4 A h_{i}}{\omega}\right)\left(g_{r} L_{r}-g_{i} L_{i}\right)
\end{array}\right], \\
& d_{11}=\left[\begin{array}{c}
-\frac{4 A h_{r} h_{i}}{\omega}\left(h_{r}-g_{r}\right)+\alpha\left(1-\frac{4 A h_{i}}{\omega}\right)\left(L_{r} g_{r}-L_{i} g_{i}\right) \\
-\alpha\left(h_{r}\left(g_{r} c_{5 r}-g_{i} c_{5 i}\right)-h_{i}\left(g_{r} c_{5 i}+g_{i} c_{5 r}\right)\right) \\
+\frac{4 \alpha A h_{r}}{\omega}\left(g_{i} L_{r}-g_{r} L_{i}\right)+\left(1-\frac{4 A h_{i}}{\omega}\right)\left(g_{r} h_{r}+g_{i} h_{i}\right) \\
-\alpha\left(M_{r} h_{r}+M_{i} h_{i}\right)
\end{array}\right], \\
& d_{12}=\left[\begin{array}{c}
-\frac{4 \alpha A h_{r}^{2}}{\omega}\left(L_{r} g_{i}-L_{i} g_{r}\right)+\alpha h_{r}\left(1-\frac{4 A h_{i}}{\omega}\right)\left(L_{r} g_{r}+L_{i} g_{i}\right) \\
-\alpha\left(h_{r}\left(g_{r} M_{r}+g_{i} M_{i}\right)-h_{i}\left(g_{r} M_{i}+g_{i} M_{r}\right)\right) \\
-\frac{4 \alpha A h_{r} h_{i}}{\omega}\left(g_{r} L_{r}-g_{i} L_{i}\right)+\alpha h_{i}\left(1-\frac{4 A h_{i}}{\omega}\right)\left(g_{i} L_{r}-g_{r} L_{i}\right)
\end{array}\right] \text {, }
\end{aligned}
$$


Rotating second-grade flow with suction

$$
\begin{aligned}
& d_{13}=\left[\begin{array}{c}
\frac{-8 A h_{r} h_{i}}{\omega}\left(h_{r}-\alpha L_{r}\right)+\alpha c_{5 r}\left(h_{r}^{2}+h_{i}^{2}\right) \\
+\frac{4 A h_{i}}{\omega}\left(h_{r}^{2}-h_{i}^{2}\right)-\frac{4 \alpha A L_{i}}{\omega}\left(h_{r}^{2}-h_{i}^{2}\right)-\alpha\left(h_{r} c_{3 r}+h_{i} c_{3 i}\right)
\end{array}\right], \\
& d_{14}=\left[\begin{array}{c}
\frac{-8 A h_{r} h_{i}}{\omega}\left(h_{r} L_{r}-h_{i} L_{i}\right)+\frac{4 \alpha A}{\omega}\left(h_{r}^{2}-h_{i}^{2}\right)\left(h_{r} L_{i}-h_{i} L_{r}\right) \\
+\alpha c_{3 r}\left(h_{r}^{2}+h_{i}^{2}\right)
\end{array}\right], \\
& d_{15}=\left[\begin{array}{c}
\frac{4 A h_{r}}{\omega}\left(g_{r}^{2}-g_{i}^{2}\right)\left(h_{r}-\alpha L_{r}\right)+2 g_{r} g_{i}\left(1-\frac{4 A h_{i}}{\omega}\right)\left(h_{r}-\alpha L_{r}\right) \\
-2 \alpha\left(h_{r}\left(g_{r} M_{i}-g_{i} M_{r}\right)-h_{i}\left(g_{r} M_{r}-g_{i} M_{i}\right)\right) \\
-\alpha\left(g_{r}^{2}-g_{i}^{2}\right)\left(h_{r} c_{5 i}-h_{i} c_{5 r}\right) \\
-\left(1-\frac{4 A h_{i}}{\omega}\right)\left(g_{r}^{2}-g_{i}^{2}\right)\left(h_{i}-\alpha L_{i}\right) \\
-2 \alpha g_{r} g_{i}\left(h_{r} c_{5 r}+h_{i} c_{5 i}\right)+\frac{8 A h_{r} g_{r} g_{i}}{\omega}\left(h_{i}-\alpha L_{i}\right)
\end{array}\right], \\
& d_{16}=\left[\begin{array}{c}
\frac{4 \alpha A h_{r}}{\omega}\left(g_{r}^{2}-g_{i}^{2}\right)\left(h_{r} L_{r}-h_{i} L_{i}\right)+2 \alpha g_{r} g_{i}\left(h_{r} M_{r}+g_{i} M_{i}\right) \\
+\alpha\left(g_{r}^{2}-g_{i}^{2}\right)\left(h_{r} M_{i}-h_{i} M_{r}\right)+\frac{8 \alpha A h_{r} g_{r} g_{i}}{\omega}\left(h_{r} L_{i}+h_{i} L_{r}\right) \\
-\alpha\left(1-\frac{4 A h_{i}}{\omega}\right)\left(g_{r}^{2}-g_{i}^{2}\right)\left(h_{r} L_{i}+h_{i} L_{r}\right) \\
+2 \alpha g_{r} g_{i}\left(1-\frac{4 A h_{i}}{\omega}\right)\left(h_{r} L_{r}-h_{i} L_{i}\right)
\end{array}\right], \\
& d_{17}=\left[\begin{array}{c}
-\frac{4 A h_{r}}{\omega}\left(g_{r}^{2}-g_{i}^{2}\right)\left(h_{i}-\alpha L_{i}\right)+2 g_{r} g_{i}\left(1-\frac{4 A h_{i}}{\omega}\right)\left(h_{i}-\alpha L_{i}\right) \\
+2 \alpha\left(h_{r}\left(g_{r} M_{r}-g_{i} M_{i}\right)+h_{i}\left(g_{r} M_{i}-g_{i} M_{r}\right)\right) \\
+\alpha\left(g_{r}^{2}-g_{i}^{2}\right)\left(h_{r} c_{5 r}+h_{i} c_{5 i}\right)+\frac{8 A h_{r} g_{r} g_{i}}{\omega}\left(h_{r}+\alpha L_{r}\right) \\
-\left(1-\frac{4 A h_{i}}{\omega}\right)\left(g_{r}^{2}-g_{i}^{2}\right)\left(h_{r}-\alpha L_{r}\right)-2 \alpha g_{r} g_{i}\left(h_{r} c_{5 i}-h_{i} c_{5 r}\right)
\end{array}\right], \\
& d_{18}=\left[\begin{array}{c}
-\frac{4 \alpha A h_{r}}{\omega}\left(g_{r}^{2}-g_{i}^{2}\right)\left(h_{r} L_{i}+h_{i} L_{r}\right)+\frac{8 \alpha A h_{r} g_{r} g_{i}}{\omega}\left(h_{r} L_{r}-h_{i} L_{i}\right) \\
-2 \alpha g_{r} g_{i}\left(1-\frac{4 A h_{i}}{\omega}\right)\left(h_{r} L_{i}+h_{i} L_{r}\right) \\
+2 \alpha g_{r} g_{i}\left(h_{r} M_{i}-h_{i} M_{r}\right)-\alpha\left(g_{r}^{2}-g_{i}^{2}\right)\left(h_{r} M_{r}+h_{i} M_{i}\right) \\
-\alpha\left(1-\frac{4 A h_{i}}{\omega}\right)\left(g_{r}^{2}-g_{i}^{2}\right)\left(h_{r} L_{r}-h_{i} L_{i}\right)
\end{array}\right] \text {, } \\
& d_{19}=\left[\begin{array}{c}
\frac{8 A h_{r} h_{i}}{\omega}\left(h_{r}^{2}+h_{i}^{2}\right)-\alpha\left(h_{r}^{3} c_{5 r}+h_{i}^{3} c_{5 i}\right)+\alpha h_{r}^{2} h_{i} c_{5 i} \\
-\frac{12 \alpha A h_{r} h_{i}}{\omega}\left(h_{r} L_{r}-h_{i} L_{i}\right)+\frac{4 \alpha A}{\omega}\left(L_{r} h_{i}^{3}-L_{i} h_{r}^{3}\right) \\
+2 \alpha c_{3 r}\left(h_{r}^{2}+h_{i}^{2}\right)+\alpha h_{r} h_{i}\left(h_{r} c_{5 i}-h_{i} c_{5 r}\right)
\end{array}\right],
\end{aligned}
$$


T. Hayat et al. 569

$$
\begin{aligned}
& d_{20}=\left[\begin{array}{c}
\frac{8 \alpha A h_{r} h_{i} L_{r}}{\omega}\left(h_{r}^{2}+h_{i}^{2}\right)-\frac{4 \alpha A L_{i}}{\omega}\left(h_{r}^{4}-h_{i}^{4}\right) \\
-\alpha\left(h_{r}^{3} c_{3 r}+h_{i}^{3} c_{3 i}\right)+\alpha h_{r} h_{i}\left(h_{r} c_{3 i}-h_{i} c_{3 r}\right)
\end{array}\right], \\
& d_{21}=\left[\begin{array}{c}
g_{i}\left(1-\frac{4 A h_{i}}{\omega}\right)\left(h_{r}^{2}-h_{i}^{2}\right)-\alpha h_{r}^{2}\left(g_{r} c_{5 i}+g_{i} c_{5 r}\right)+2 \alpha h_{r} h_{i} M_{r} \\
+2 \alpha h_{r} h_{i}\left(g_{r} c_{5 r}-g_{i} c_{5 i}\right)-2 h_{r} g_{r}\left(1-\frac{4 A h_{i}}{\omega}\right)\left(h_{i}-\alpha L_{i}\right) \\
+\frac{4 A h_{r} g_{r}}{\omega}\left(h_{r}^{2}-h_{i}^{2}\right)+\alpha h_{i}^{2}\left(g_{r} c_{5 i}+g_{i} c_{5 r}\right)-\frac{8 \alpha A h_{r}^{2} g_{r} L_{r}}{\omega} \\
+\frac{8 A h_{r}^{2} g_{i}}{\omega}\left(h_{i}-\alpha L_{i}\right)-2 \alpha L_{r}\left(1-\frac{4 A h_{i}}{\omega}\right)\left(h_{r} g_{i}-h_{i} g_{r}\right) \\
-\frac{8 \alpha A h_{r} h_{i}}{\omega}\left(L_{r} g_{i}-L_{i} g_{r}\right)+2 \alpha L_{i} h_{i} g_{i}\left(1-\frac{4 A h_{i}}{\omega}\right)
\end{array}\right], \\
& d_{22}=\left[\begin{array}{c}
\alpha\left(g_{r} M_{i}+g_{i} M_{r}\right)\left(h_{r}^{2}-h_{i}^{2}\right) \\
-\frac{8 \alpha A h_{r}}{\omega}\left(h_{r}^{2}-h_{i}^{2}\right)\left(L_{r} g_{r}+L_{i} g_{i}\right) \\
+2 \alpha h_{r} h_{i}\left(g_{r} M_{r}-g_{i} M_{i}\right)+\frac{8 \alpha A h_{r} h_{i}}{\omega}\left(L_{r} g_{i}-L_{i} g_{r}\right) \\
+\alpha\left(1-\frac{4 A h_{i}}{\omega}\right)\left(L_{r} g_{i}-L_{i} g_{r}\right)\left(h_{r}^{2} g_{i}-h_{i}^{2}\right) \\
-2 \alpha h_{r} h_{i}\left(1-\frac{4 A h_{i}}{\omega}\right)\left(L_{r} g_{r}+L_{i} g_{i}\right)
\end{array}\right], \\
& d_{23}=\left[\begin{array}{c}
-g_{r}\left(1-\frac{4 A h_{i}}{\omega}\right)\left(h_{r}^{2}-h_{i}^{2}\right)+\alpha g_{r} c_{5 r}\left(h_{r}^{2}-h_{i}^{2}\right) \\
+\alpha g_{i} c_{5 i}\left(h_{r}^{2}+h_{i}^{2}\right)-\frac{8 A h_{r}^{2} g_{r}}{\omega}\left(h_{i}-\alpha L_{i}\right) \\
+\frac{4 A h_{r} g_{i}}{\omega}\left(h_{r}^{2}-h_{i}^{2}\right)+2 \alpha h_{r} h_{i} M_{i} \\
+2 \alpha h_{r} h_{i}\left(g_{r} c_{5 i}+g_{i} c_{5 r}\right) \\
-2 h_{r} g_{i}\left(1-\frac{4 A h_{i}}{\omega}\right)\left(h_{i}+\alpha L_{i}\right) \\
-\frac{8 \alpha A h_{r}^{2} g_{i} L_{r}}{\omega}+\frac{8 \alpha A h_{r} h_{i}}{\omega}\left(L_{r} g_{r}+L_{i} h_{i}\right) \\
+2 \alpha h_{i}\left(1-\frac{4 A h_{i}}{\omega}\right)\left(L_{r} g_{i}-L_{i} g_{r}\right)
\end{array}\right], \\
& d_{24}=\left[\begin{array}{c}
-\alpha\left(1-\frac{4 A h_{i}}{\omega}\right)\left(h_{r}^{2}-h_{i}^{2}\right)\left(L_{r} g_{r}+L_{i} g_{i}\right) \\
-\alpha\left(h_{r}^{2}-h_{i}^{2}\right)\left(M_{r} g_{r}-M_{i} g_{i}\right)-\frac{8 \alpha A h_{r}^{2} h_{i}}{\omega}\left(L_{r} g_{r}+L_{i} g_{i}\right) \\
+\frac{4 \alpha A h_{r}}{\omega}\left(L_{r} g_{i}-L_{i} g_{r}\right)\left(h_{r}^{2}-h_{i}^{2}\right) \\
-2 \alpha h_{r} h_{i}\left(1-\frac{4 A h_{i}}{\omega}\right)\left(L_{r} g_{i}-L_{i} g_{r}\right) \\
-2 \alpha h_{r} h_{i}\left(M_{i} g_{r}+M_{r} g_{i}\right)
\end{array}\right],
\end{aligned}
$$


Rotating second-grade flow with suction

$$
\begin{aligned}
& d_{25}=\left[\begin{array}{c}
-\alpha\left(h_{r}^{3} c_{5 r}+h_{i}^{3} c_{5 i}\right)+\alpha c_{3 r}\left(h_{r}^{2}-h_{i}^{2}\right)+\frac{8 \alpha A h_{r} L_{i}}{\omega}\left(h_{r}^{2}-h_{i}^{2}\right) \\
+2 \alpha h_{r} h_{i} c_{3 i}-\alpha h_{r} h_{i}\left(h_{r} c_{5 i}+h_{i} c_{5 r}\right)-\frac{8 \alpha A h_{i}^{3} L_{r}}{\omega}
\end{array}\right], \\
& d_{26}=\left[\begin{array}{c}
-\frac{4 \alpha A L_{i}}{\omega}\left(h_{r}^{4}+h_{i}^{4}\right)-\frac{8 \alpha A h_{i}^{2} L_{i} h_{r}^{2}}{\omega} \\
-\alpha\left(h_{r}^{3} c_{3 r}+h_{i}^{3} c_{3 i}\right)-\alpha h_{r} h_{i}\left(h_{r} c_{3 i}+h_{i} c_{3 r}\right)
\end{array}\right], \\
& d_{27}=\left[-h_{r}^{3}+3 \alpha h_{r}^{2} L_{r}-h_{r} h_{i}^{2}+2 \alpha h_{r} h_{i} L_{i}+\alpha L_{r} h_{i}^{2}\right] \text {, } \\
& d_{28}=-2 \alpha L_{r} h_{r}\left(h_{r}^{2}+h_{i}^{2}\right), \\
& m_{1}=m_{1 r}+i m_{1 i}=\left[\begin{array}{c}
-P_{r} A\left(P_{r}\left(1+d_{7}\right)-2 h_{r} d_{7}+d_{8}\right)+P A d_{27} \\
-\left(2 E_{c}+i \omega P\right) d_{13}+P\left(d_{19}+d_{25}\right)
\end{array}\right] \text {, } \\
& m_{2}=m_{2 r}+i m_{2 i}=\left[\begin{array}{c}
2 P_{r} A h_{r} d_{8}-\left(2 E_{c}+i \omega P\right) d_{14} \\
+P A d_{28}+P\left(d_{20}+d_{26}\right)
\end{array}\right] \text {, } \\
& m_{3}=m_{3 r}+i m_{3 i}=\left[-\left(2 E_{c}+i \omega P\right) d_{9}+P\left(d_{15}+d_{21}\right)\right] \text {, } \\
& m_{4}=m_{4 r}+i m_{4 i}=\left[-\left(2 E_{c}+i \omega P\right) d_{10}+P\left(d_{16}+d_{22}\right)\right] \text {, } \\
& m_{5}=m_{5 r}+i m_{5 i}=\left[-\left(2 E_{c}+i \omega P\right) d_{11}+P\left(d_{17}+d_{23}\right)\right] \text {, } \\
& m_{6}=m_{6 r}+i m_{6 i}=\left[-\left(2 E_{c}+i \omega P\right) d_{12}+P\left(d_{18}+d_{24}\right)\right] \text {, } \\
& m_{7}=m_{7 r}+i m_{7 i} \text {, } \\
& m_{7 r}=\frac{m_{1 r}\left(4 h_{r}^{2}-2 h_{r} P_{r}\right)-m_{1 i}\left(\omega P_{r} / 4\right)}{\left(4 h_{r}^{2}-2 h_{r} P_{r}\right)^{2}+\left(\omega P_{r} / 4\right)^{2}}, \quad m_{7 i}=\frac{m_{1 i}\left(4 h_{r}^{2}-2 h_{r} P_{r}\right)+m_{1 r}\left(\omega P_{r} / 4\right)}{\left(4 h_{r}^{2}-2 h_{r} P_{r}\right)^{2}+\left(\omega P_{r} / 4\right)^{2}}, \\
& m_{8}=m_{8 r}+i m_{8 i} \\
& m_{8 r}=\frac{m_{2 r}\left(4 h_{r}^{2}-2 h_{r} P_{r}\right)-m_{2 i}\left(\omega P_{r} / 4\right)}{\left(4 h_{r}^{2}-2 h_{r} P_{r}\right)^{2}+\left(\omega P_{r} / 4\right)^{2}}, \quad m_{8 i}=\frac{m_{2 i}\left(4 h_{r}^{2}-2 h_{r} P_{r}\right)+m_{2 r}\left(\omega P_{r} / 4\right)}{\left(4 h_{r}^{2}-2 h_{r} P_{r}\right)^{2}+\left(\omega P_{r} / 4\right)^{2}}, \\
& n_{1}=n_{1 r}+i n_{1 i}=\left[\begin{array}{l}
\left(4 h_{r}^{2}-2 h_{r} P_{r}\right)^{2}+\left(\frac{\omega P_{r}}{4}\right)^{2} \\
-2 i\left(4 h_{r}^{2}-2 h_{r} P_{r}\right)\left(\frac{\omega P_{r}}{4}\right)
\end{array}\right], \\
& n_{1 r}=\left(4 h_{r}^{2}-2 h_{r} P_{r}\right)^{2}+\left(\frac{\omega P_{r}}{4}\right)^{2}, \quad n_{1 i}=-2\left(4 h_{r}^{2}-2 h_{r} P_{r}\right)\left(\frac{\omega P_{r}}{4}\right), \\
& m_{9}=m_{9 r}+i m_{9 i} \text {, } \\
& m_{9 r}=\frac{\left(4 h_{r}-P_{r}\right)\left(n_{1 r} m_{2 r}-m_{2 i} n_{1 i}\right)}{n_{1 r}^{2}+n_{1 i}^{2}}, \quad m_{9 i}=\frac{\left(4 h_{r}-P_{r}\right)\left(n_{1 i} m_{2 r}+m_{2 i} n_{1 r}\right)}{n_{1 r}^{2}+n_{1 i}^{2}}, \\
& n_{2}=n_{2 r}+i n_{2 i}=\left[\begin{array}{c}
\left(h_{r}+g_{r}\right)^{2}-\left(h_{i}-g_{i}\right)^{2}-P_{r}\left(h_{r}+g_{r}\right) \\
+i\left(-2\left(h_{r}+g_{r}\right)\left(h_{i}-g_{i}\right)+P_{r}\left(h_{i}-g_{i}\right)-\frac{\omega P_{r}}{4}\right)
\end{array}\right], \\
& n_{2 r}=\left[\left(h_{r}+g_{r}\right)^{2}-\left(h_{i}-g_{i}\right)^{2}-P_{r}\left(h_{r}+g_{r}\right)\right] \text {, } \\
& n_{2 i}=-2\left(h_{r}+g_{r}\right)\left(h_{i}-g_{i}\right)+P_{r}\left(h_{i}-g_{i}\right)-\frac{\omega P_{r}}{4} \text {, }
\end{aligned}
$$


T. Hayat et al. 571

$$
\begin{aligned}
& m_{10}=m_{10 r}+i m_{10 i}, \quad m_{10 r}=\frac{\left(n_{2 r} m_{5 r}-m_{3 r} n_{2 i}\right)}{n_{2 r}^{2}+n_{2 i}^{2}} \\
& m_{10 i}=\frac{\left(n_{2 r} m_{5 i}-m_{3 i} n_{2 i}\right)}{n_{2 r}^{2}+n_{2 i}^{2}}, \quad m_{11}=m_{11 r}+i m_{11 i}, \\
& m_{11 r}=\frac{\left(n_{2 i} m_{5 r}+m_{3 r} n_{2 r}\right)}{n_{2 r}^{2}+n_{2 i}^{2}}, \quad m_{11 i}=\frac{\left(n_{2 i} m_{5 i}+m_{3 i} n_{2 r}\right)}{n_{2 r}^{2}+n_{2 i}^{2}}, \\
& m_{12}=m_{12 r}+i m_{12 i}, \quad m_{12 r}=\frac{\left(n_{2 r} m_{6 r}-m_{4 r} n_{2 i}\right)}{n_{2 r}^{2}+n_{2 i}^{2}}, \\
& m_{12 i}=\frac{\left(n_{2 r} m_{6 i}-m_{4 i} n_{2 i}\right)}{n_{2 r}^{2}+n_{2 i}^{2}}, \quad m_{13}=m_{13 r}+i m_{13 i}, \\
& m_{13 r}=\frac{\left(n_{2 i} m_{6 r}+m_{6 r} n_{2 r}\right)}{n_{2 r}^{2}+n_{2 i}^{2}}, \quad m_{13 i}=\frac{\left(n_{2 i} m_{6 i}+m_{6 i} n_{2 r}\right)}{n_{2 r}^{2}+n_{2 i}^{2}}, \\
& n_{3}=n_{3 r}+i n_{3 i}=\left[\left(2\left(h_{r}+g_{r}\right)-P_{r}\right)+i\left(-2\left(h_{i}-g_{i}\right)\right)\right], \\
& n_{3 r}=2\left(h_{r}+g_{r}\right)-P_{r}, \quad n_{3 i}=-2\left(h_{i}-g_{i}\right), \quad n_{4}=n_{4 r}+i n_{4 i} \text {, } \\
& n_{4 r}=\left[\begin{array}{c}
\left(h_{r}+g_{r}\right)^{4}+\left(h_{i}-g_{i}\right)^{4}+\frac{\omega P_{r}^{2}}{2}\left(h_{i}-g_{i}\right) \\
-6\left(h_{r}+g_{r}\right)^{2}\left(h_{i}-g_{i}\right)^{2}+\frac{\omega^{2} P_{r}^{2}}{16} \\
+P_{r}^{2}\left(\left(h_{r}+g_{r}\right)^{2}-\left(h_{i}-g_{i}\right)^{2}\right)-\omega P_{r}\left(h_{r}+g_{r}\right)\left(h_{i}-g_{i}\right) \\
+2 P_{r}\left(-\left(h_{r}+g_{r}\right)^{3}+3\left(h_{r}+g_{r}\right)\left(h_{i}-g_{i}\right)^{2}\right)
\end{array}\right], \\
& n_{4 i}=\left[\begin{array}{c}
-4\left(h_{r}+g_{r}\right)^{3}\left(h_{i}-g_{i}\right)+4\left(h_{r}+g_{r}\right)\left(h_{i}-g_{i}\right)^{3} \\
+2 P_{r}\left(3\left(h_{r}+g_{r}\right)^{2}\left(h_{i}-g_{i}\right)-\left(h_{i}-g_{i}\right)^{3}\right)-\frac{\omega P_{r}^{2}}{2}\left(h_{r}+g_{r}\right) \\
-2 P_{r}^{2}\left(h_{r}+g_{r}\right)\left(h_{i}-g_{i}\right)-\frac{\omega P_{r}}{2}\left(\left(h_{r}+g_{r}\right)^{2}-\left(h_{i}-g_{i}\right)^{2}\right)
\end{array}\right] \text {, } \\
& m_{14}=m_{14 r}+i m_{14 i}, \\
& m_{14 r}=\frac{m_{6 r}\left(n_{4 r} n_{3 r}+n_{4 i} n_{3 i}\right)+m_{4 r}\left(n_{4 r} n_{3 i}-n_{4 i} n_{3 r}\right)}{n_{4 r}^{2}+n_{4 i}^{2}}, \\
& m_{14 i}=\frac{m_{6 i}\left(n_{4 r} n_{3 r}+n_{4 i} n_{3 i}\right)+m_{4 i}\left(n_{4 r} n_{3 i}-n_{4 i} n_{3 r}\right)}{n_{4 r}^{2}+n_{4 i}^{2}}, \\
& m_{15}=m_{15 r}+i m_{15 i}, \\
& m_{15 r}=\frac{-m_{6 r}\left(n_{4 r} n_{3 i}-n_{4 i} n_{3 r}\right)+m_{4 r}\left(n_{4 r} n_{3 r}+n_{4 i} n_{3 i}\right)}{n_{4 r}^{2}+n_{4 i}^{2}}, \\
& m_{14 i}=\frac{-m_{6 i}\left(n_{4 r} n_{3 i}-n_{4 i} n_{3 r}\right)+m_{4 i}\left(n_{4 r} n_{3 r}+n_{4 i} n_{3 i}\right)}{n_{4 r}^{2}+n_{4 i}^{2}}, \\
& m_{16}=m_{16 r}+i m_{16 i}=m_{7}+m_{9}+m_{10}+m_{14}, \\
& m_{16 r}=m_{7 r}+m_{9 r}+m_{10 r}+m_{146 r}, \quad m_{16 i}=m_{7 i}+m_{9 i}+m_{10 i}+m_{146 i} \text {, } \\
& f=f_{r}+i f_{i}=\frac{\left(P_{r}+\sqrt{P_{r}^{2}+i \omega P_{r}}\right)}{2},
\end{aligned}
$$




$$
\begin{gathered}
f_{r}=\frac{P_{r}}{2}+\frac{a_{2}}{2}=\frac{P_{r}}{2}+\frac{1}{2}\left[\frac{P_{r}^{2}+\sqrt{P_{r}^{4}+\omega^{2} P_{r}^{2}}}{2}\right]^{1 / 2}, \\
f_{i}=\frac{b_{2}}{2}=\frac{1}{2}\left[\frac{-P_{r}^{2}+\sqrt{P_{r}^{4}+\omega^{2} P_{r}^{2}}}{2}\right]^{1 / 2}, \\
a_{2}=\left[\frac{P_{r}^{2}+\sqrt{P_{r}^{4}+\omega^{2} P_{r}^{2}}}{2}\right]^{1 / 2}, \quad b_{2}=\left[\frac{-P_{r}^{2}+\sqrt{P_{r}^{4}+\omega^{2} P_{r}^{2}}}{2}\right]^{1 / 2}, \\
r_{2}=a_{2}^{2}+b_{2}^{2}=\sqrt{P_{r}^{4}+\omega^{2} P_{r}^{2}} .
\end{gathered}
$$

From (3.24), (3.28), and (3.29), we can write

$$
\theta=\theta_{0}+\epsilon\left(\theta_{1 r} \cos \omega t-\theta_{1 i} \sin \omega t\right)
$$

in which

$$
\begin{aligned}
\theta_{1 r}= & -e^{-f_{r} \eta}\left(m_{16 r} \cos f_{i} \eta-m_{16 i} \sin f_{i} \eta\right)+\left(m_{7 r}+m_{9 r}+m_{8 r} \eta\right) e^{-2 h_{r} \eta} \\
& +\left[\begin{array}{c}
\left(m_{10 r}+m_{14 r}+m_{12 r} \eta\right) \cos \left(h_{i}-g_{i}\right) \eta \\
+\left(m_{11 r}+m_{15 r}+m_{13 r} \eta\right) \sin \left(h_{i}-g_{i}\right) \eta
\end{array}\right] e^{-\left(h_{r}+g_{r}\right) \eta}, \\
\theta_{1 i}= & -e^{-f_{r} \eta}\left(m_{16 r} \sin f_{i} \eta+m_{16 i} \cos f_{i} \eta\right)+\left(m_{7 i}+m_{9 i}+m_{8 i} \eta\right) e^{-2 h_{r} \eta} \\
& +\left[\begin{array}{c}
\left(m_{10 i}+m_{14 i}+m_{12 i} \eta\right) \cos \left(h_{i}-g_{i}\right) \eta \\
+\left(m_{11 i}+m_{15 i}+m_{13 i} \eta\right) \sin \left(h_{i}-g_{i}\right) \eta
\end{array}\right] e^{-\left(h_{r}+g_{r}\right) \eta} .
\end{aligned}
$$

\section{Discussion of results}

In this paper, we consider the problem of heat transfer in rotating flow of an incompressible fluid of second grade. A perturbation procedure has been used to obtain the analytic solution. The effects of various parameters such as $\Omega, P_{r}$, and $E_{c}$ on the real and imaginary parts of velocity $(u, v)$ and temperature $\left(\theta_{r}, \theta_{i}\right)$ distributions are studied and the results have been presented by several graphs.

To study the effect of $\Omega$ on the velocity components, we have plotted $u$ and $v$ against $\eta$ in Figures 4.1, and 4.2 for Newtonian and second-grade fluids. From Figure 4.1(a), it is observed that near the plate $u$ increases with the increase of $\Omega$. Figure 4.1(b) indicates that $u$ increases very near to the plate and then fluctuates through an increase in $\Omega$. The comparison of these two figures reveals that $u$ in case of second-grade fluid is greater than that of Newtonian fluid. Also, the velocity boundary layer thickness for second-grade fluid is larger than the Newtonian fluid. It is also seen from Figures 4.2(a) and 4.2(b) that $v$ increases near the plate and then decreases for large value of $\Omega$. The fluctuations in second-grade fluid are more visible than that of Newtonian fluid. Also, the value of $v$ for second-grade fluid is smaller than in the case of Newtonian fluid.

Figures 4.3 and 4.4 show the effect of $\Omega$ on the real $\left(\theta_{r}\right)$ and imaginary $\left(\theta_{i}\right)$ parts of temperature distributions. Figure 4.3(a) shows that with the increase of $\Omega, \theta_{r}$ decreases near the wall. As shown in Figure 4.3(b), we can see that as $\Omega$ increases, $\theta_{r}$ increases near 
T. Hayat et al. 573

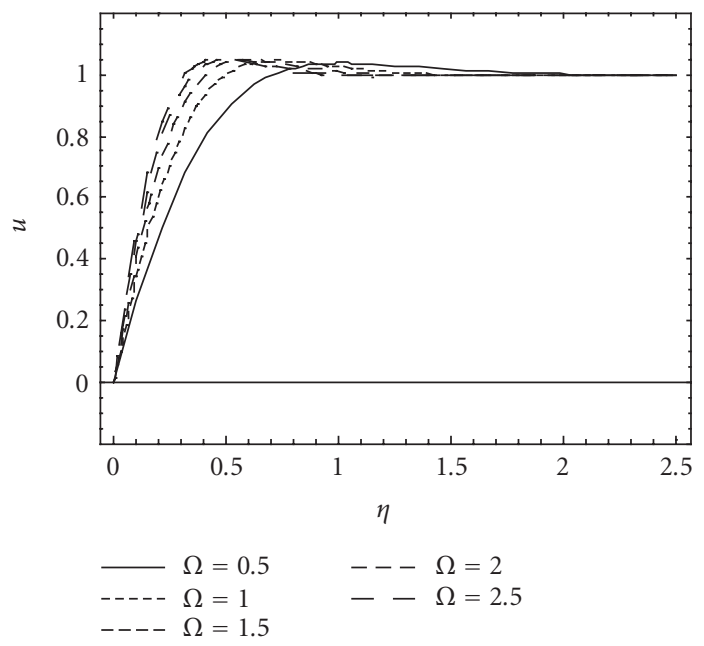

(a)

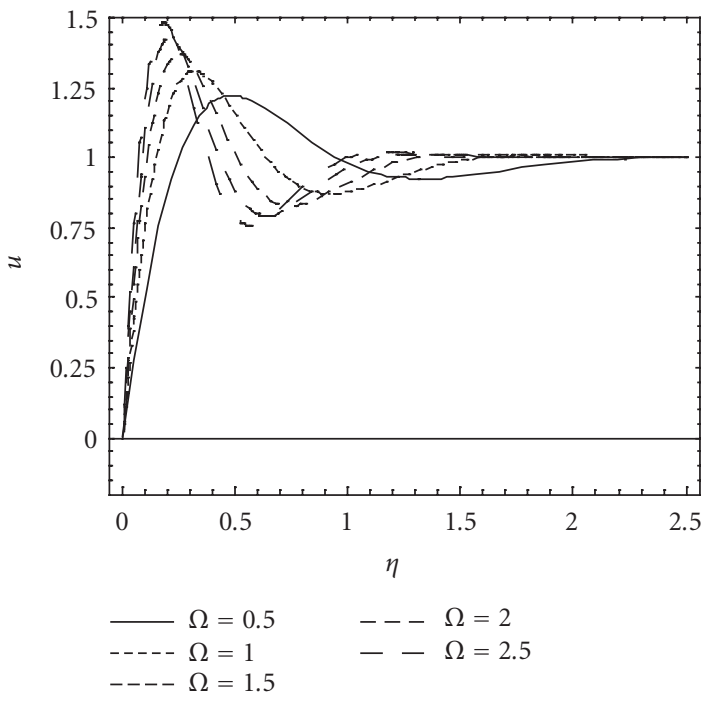

(b)

Figure 4.1. Effect of $\Omega$ on real part of velocity profile $u$ versus $\eta$. In (a) for Newtonian fluid at $\alpha=0$, $\omega t=\pi / 2, A=0.2, \epsilon=\omega=0.5, W_{0}=-0.5, \nu=0.1$. In (b) for second-grade fluid at $\alpha=0.4$. 
574 Rotating second-grade flow with suction

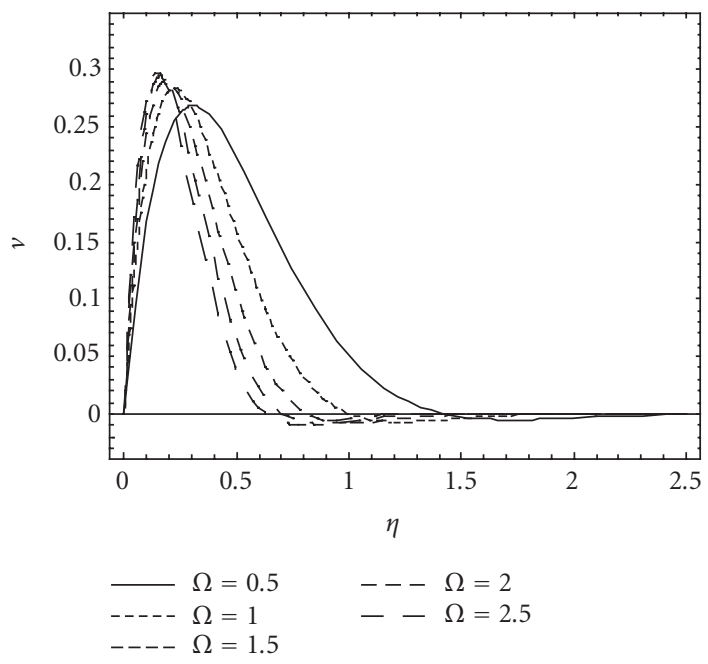

(a)

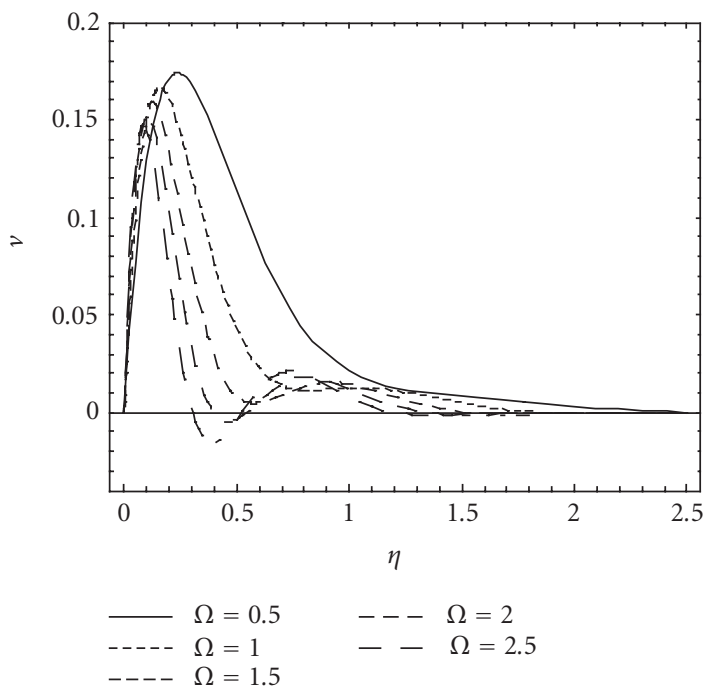

(b)

Figure 4.2. Effect of $\Omega$ on imaginary part of velocity profile $v$ versus $\eta$. In (a) for Newtonian fluid at $\alpha=0, \omega t=\pi / 2, A=0.2, \epsilon=\omega=0.5, W_{0}=-0.5, \nu=0.1$. In (b) for second-grade fluid at $\alpha=0.1$. 
T. Hayat et al. 575

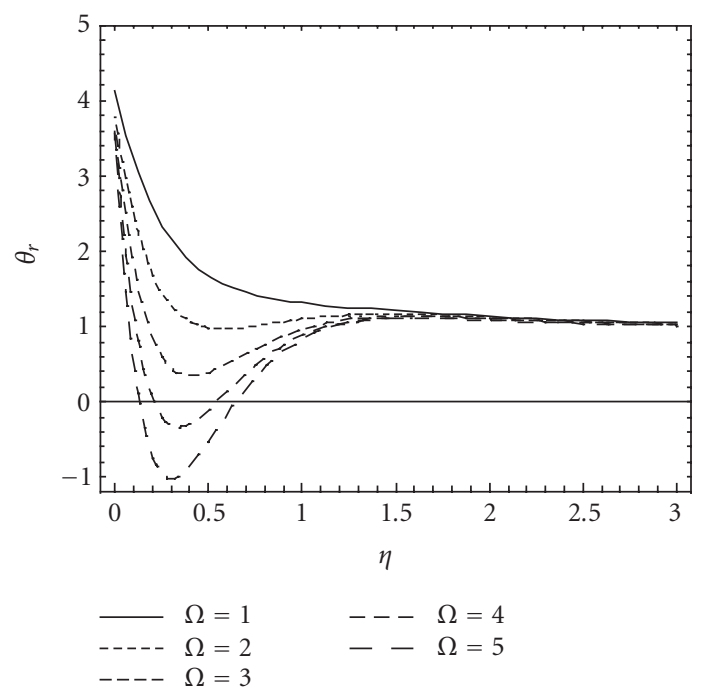

(a)

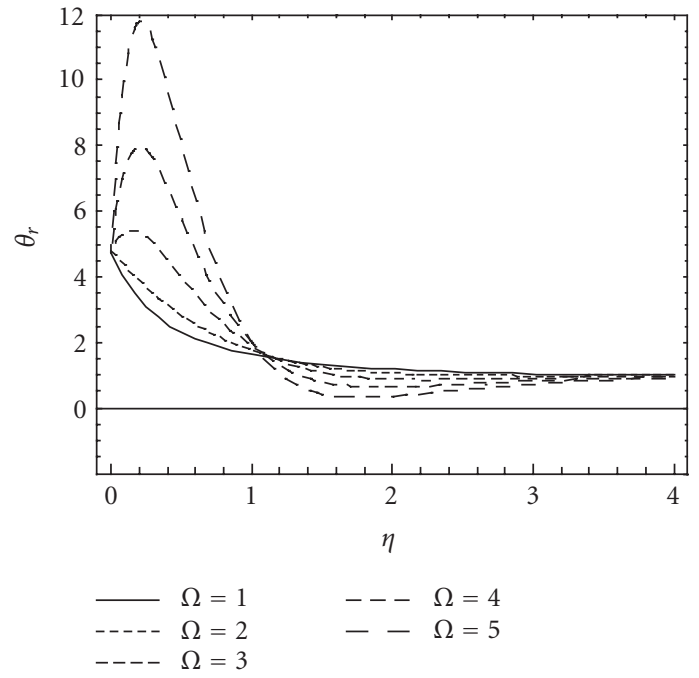

(b)

Figure 4.3. Effect of $\Omega$ on real part of temperature profile $\theta_{r}$ versus $\eta$. In (a) for Newtonian fluid at $\alpha=0, \omega t=\pi / 2, A=\epsilon=\omega=0.5, W_{0}=-0.5, \nu=0.1, P_{r}=1.5, E_{c}=5.0, k=0.2, P=0.3$. In (b) for second-grade fluid at $\alpha=0.04$. 


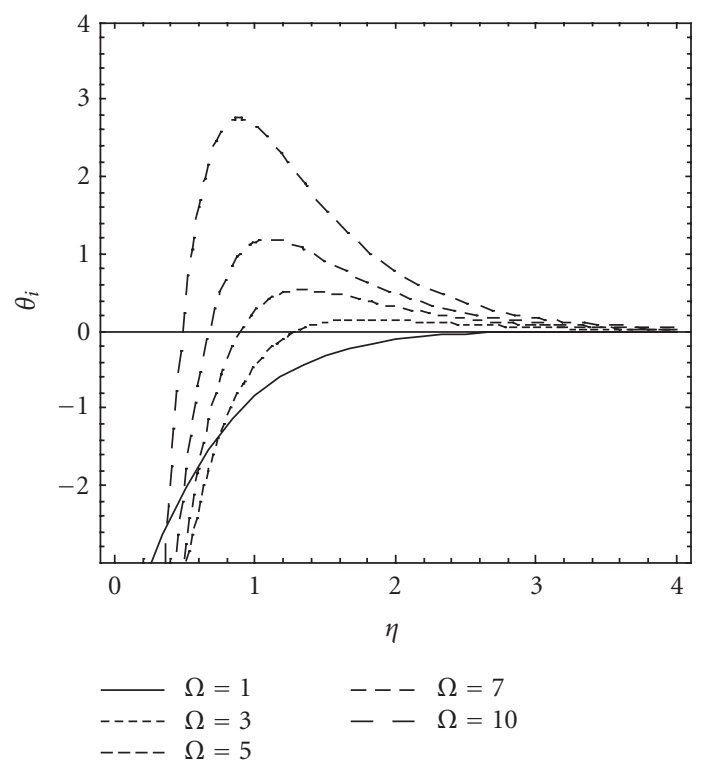

(a)

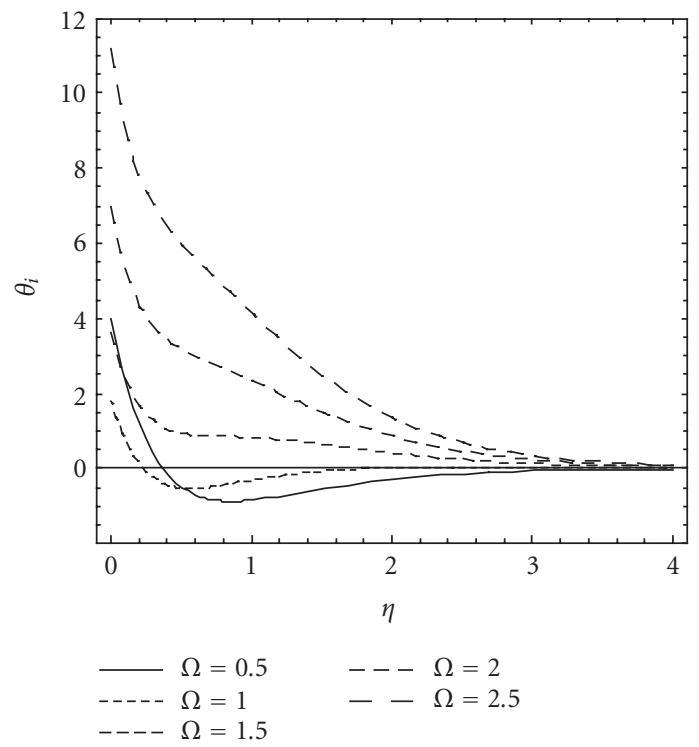

(b)

Figure 4.4. Effect of $\Omega$ on imaginary part of temperature profile $\theta_{i}$ versus $\eta$. In (a) for Newtonian fluid at $\alpha=0, \omega t=\pi / 2, A=\epsilon=\omega=0.5, W_{0}=-0.5, v=0.1, P_{r}=1.5, E_{c}=5.0, k=0.2, P=0.3$. In (b) for second-grade fluid at $\alpha=0.05$. 
T. Hayat et al. 577

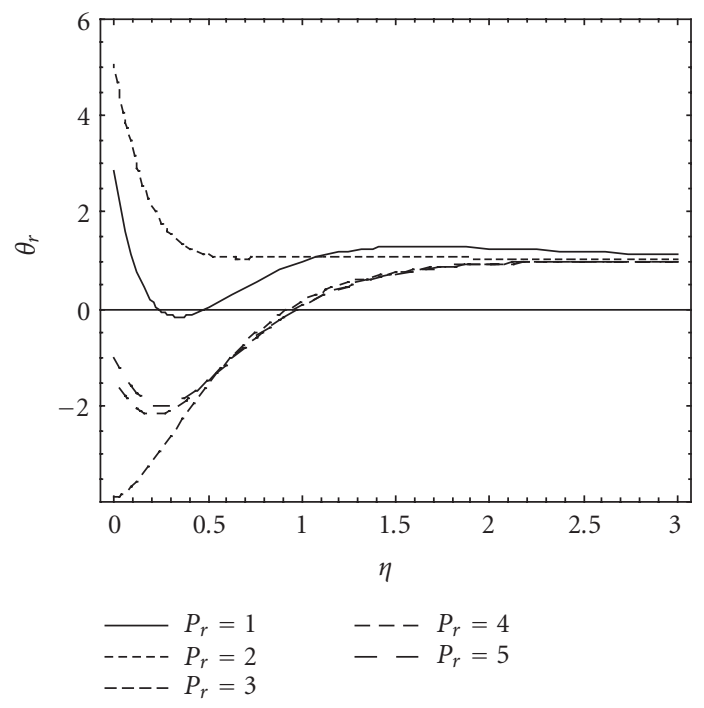

(a)

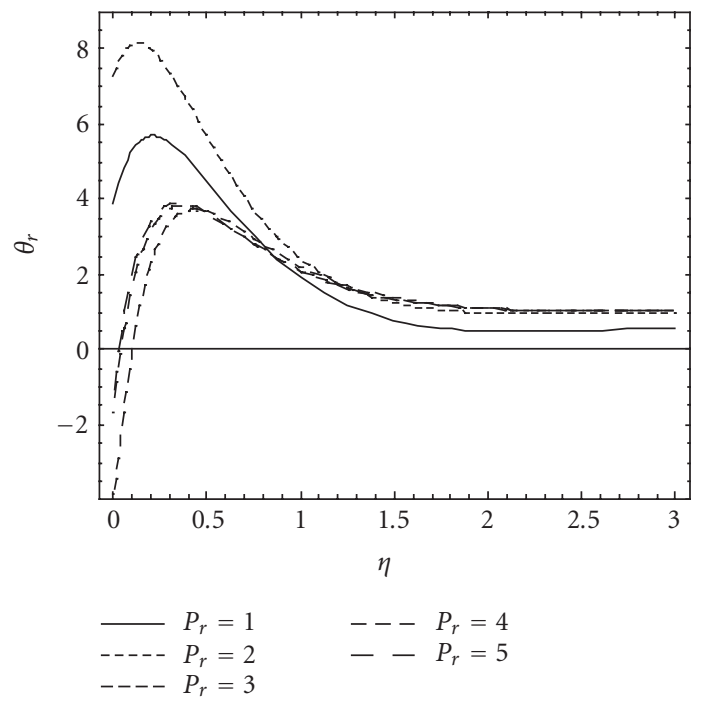

(b)

Figure 4.5. Effect of $P_{r}$ on real part of temperature profile $\theta_{r}$ versus $\eta$. In (a) for Newtonian fluid at $\alpha=0, \omega t=\pi / 2, A=\epsilon=\omega=0.5, W_{0}=-0.5, v=0.1, \Omega=3.0, E_{c}=5.0, k=0.2, P=0.3$. In (b) for second-grade fluid at $\alpha=0.05$. 


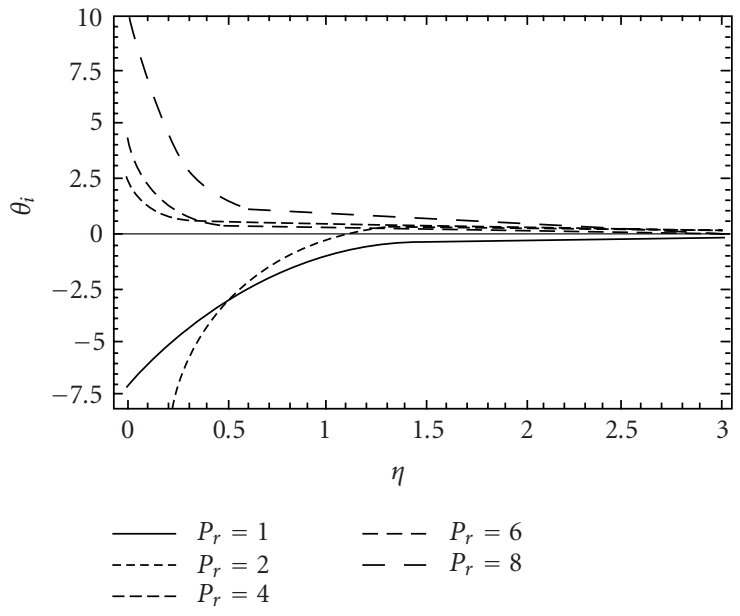

(a)

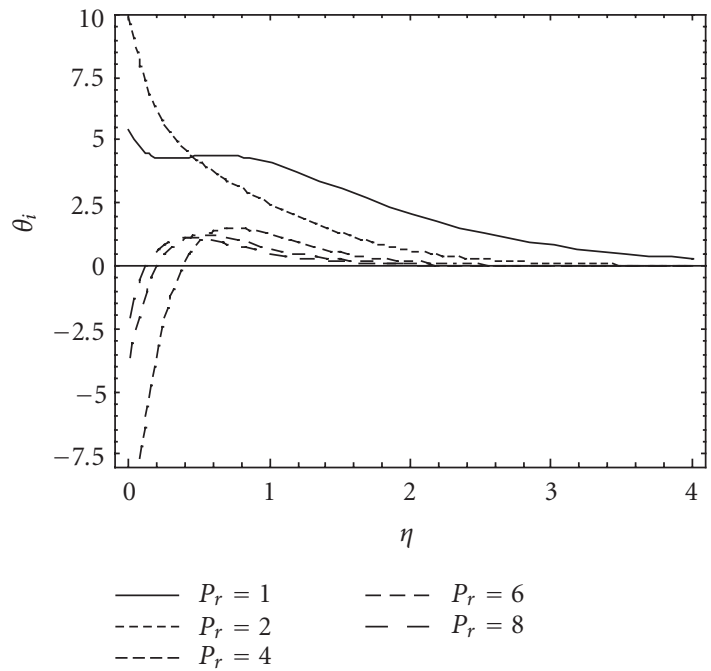

(b)

Figure 4.6. Effect of $P_{r}$ on imaginary part of temperature profile $\theta_{i}$ versus $\eta$. In (a) for Newtonian fluid at $\alpha=0, \omega t=\pi / 2, A=\epsilon=\omega=0.5, W_{0}=-0.5, \nu=0.1, \Omega=2.5, E_{c}=5.0, k=0.2, P=0.3$. In (b) for second-grade fluid at $\alpha=0.04$. 
T. Hayat et al. 579

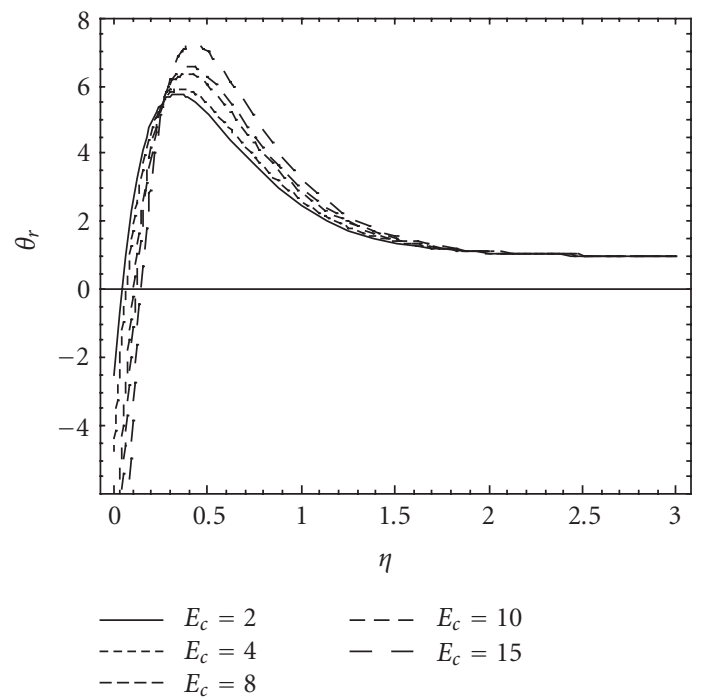

(a)

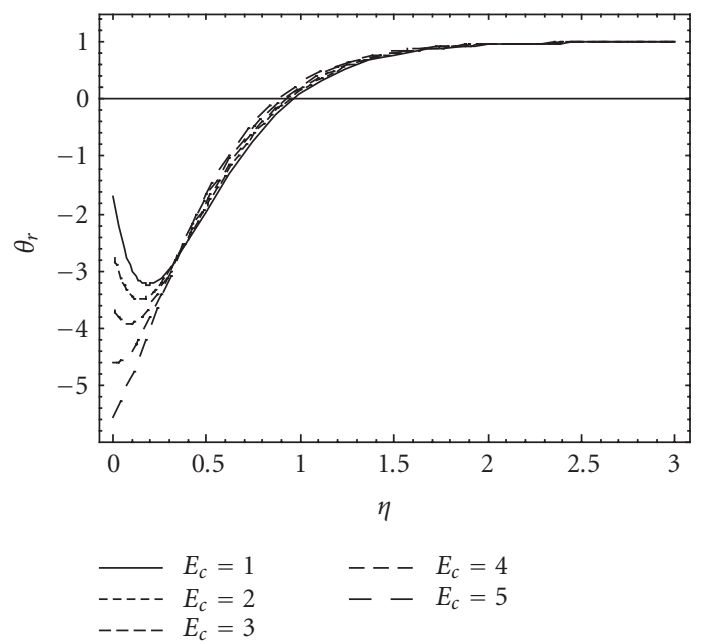

(b)

Figure 4.7. Effect of $E_{c}$ on real part of temperature profile $\theta_{r}$ versus $\eta$. In (a) for Newtonian fluid at $\alpha=0, \omega t=\pi / 2, A=\epsilon=\omega=0.5, W_{0}=-0.5, v=0.1, \Omega=4.0, P_{r}=5.0, k=0.2, P=0.3$. In (b) for second-grade fluid at $\alpha=0.04$. 


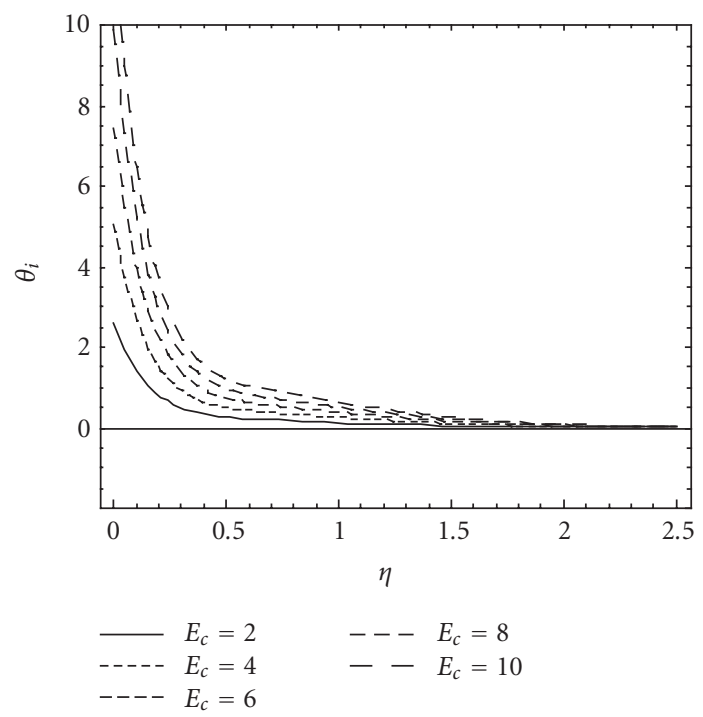

(a)

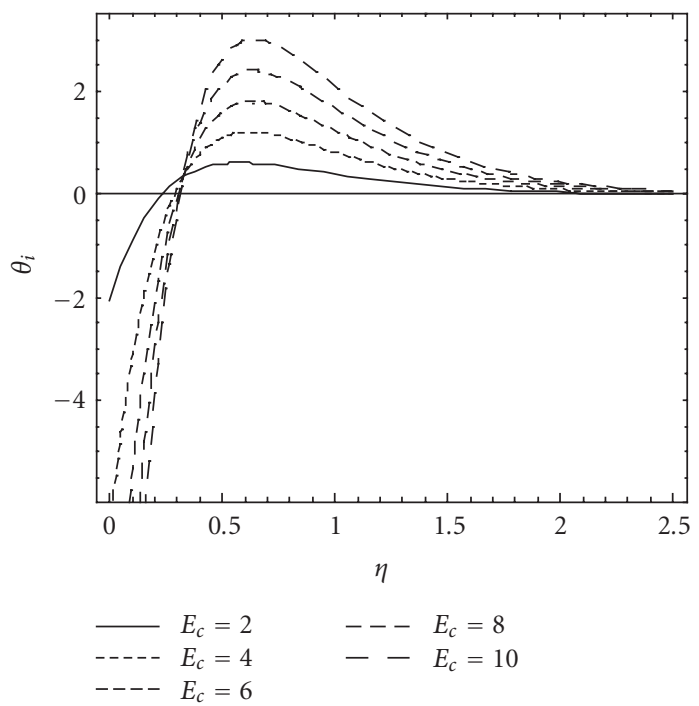

(b)

Figure 4.8. Effect of $E_{c}$ on imaginary part of temperature profile $\theta_{i}$ versus $\eta$. In (a) for Newtonian fluid at $\alpha=0, \omega t=\pi / 2, A=\epsilon=\omega=0.5, W_{0}=-0.5, v=0.1, \Omega=2.5, P_{r}=5.0, k=0.2, P=0.3$. In (b) for second-grade fluid at $\alpha=0.05$. 
the plate, and then at a distance of $\eta=1$, the $\theta_{r}$ begins to decrease. That is, the behavior of $\theta_{r}$ is quite opposite for Newtonian and second-grade fluids near the plate. Figure 4.4(a) shows the variation of $\Omega$ on $\theta_{i}$. It can be seen that as $\Omega$ increases, the value of $\theta_{i}$ decreases at a distance of approximately $\eta=0.8$ and then increases. Figure 4.4(b) indicates that $\theta_{i}$ increases near the wall for $\Omega>1$.

In order to illustrate the variation of $P_{r}$ on $\theta_{r}$ and $\theta_{i}$, we have prepared Figures 4.5 and 4.6. Figures 4.5(a) and 4.6(a) explain the effect of $P_{r}$ on $\theta_{r}$ and $\theta_{i}$, respectively, for Newtonian fluid case. From these figures, it is revealed that near the plate, $\theta_{r}$ decreases and $\theta_{i}$ increases for $P_{r}>2$. The thermal boundary layer thickness in $\theta_{r}$ increases where as for $\theta_{i}$ decreases. For second-grade fluid, we note that from Figures 4.5(b) and 4.6(b) that for $P_{r}>2, \theta_{r}$ decreases near the wall and increases far away. Also $\theta_{i}$ decreases for $P_{r}>2$.

Figures 4.7 and 4.8 show the effect of $E_{c}$ on $\theta_{r}$ and $\theta_{i}$. From Figures 4.7(a) and 4.7(b), we observe that $\theta_{r}$ decreases near the wall with the increase in $E_{c}$ and increases far away. The thermal boundary layer thickness increases for large $E_{c}$. Moreover, it can be seen from Figure 4.8(a) that $\theta_{i}$ increases for large values of $E_{c}$. From Figure 4.8(b), it can be seen that with the increase in the values of $E_{c}$, the temperature $\theta_{i}$ decreases near the plate and increases far away. The thermal boundary layer thicknesses in both fluids increases.

\section{References}

[1] R. Bandelli, Unsteady unidirectional flows of second grade fluids in domains with heated boundaries, Internat. J. Non-Linear Mech. 30 (1995), no. 3, 263-269.

[2] D. W. Beard and K. Walters, Elastico-viscous boundary-layer flows. I. Two-dimensional flow near a stagnation point, Proc. Camb. Phil. Soc. 60 (1964), 667-674.

[3] İ. T. Dolapçı and M. Pakdemirli, Approximate symmetries of creeping flow equations of a second grade fluid, Internat. J. Non-Linear Mech. 39 (2004), no. 10, 1603-1619.

[4] J. E. Dunn and R. L. Fosdick, Thermodynamics, stability, and boundedness of fluids of complexity 2 and fluids of second grade, Arch. Ration. Mech. Anal. 56 (1974), 191-252.

[5] R. L. Fosdick and K. R. Rajagopal, Anomalous features in the model of "second order fluids", Arch. Ration. Mech. Anal. 70 (1979), no. 2, 145-152.

[6] F. Franchi and B. Straughan, Stability and nonexistence results in the generalized theory of a fluid of second grade, J. Math. Anal. Appl. 180 (1993), no. 1, 122-137.

[7] G. Gupta and M. Massoudi, Flow of a generalized second grade fluid between heated plates, Acta Mech. 99 (1993), no. 1-4, 21-33.

[8] T. Hayat, Q. Abbas, S. Asghar, A. M. Siddiqui, T. Farid, and G. Murtaza, Flow of an elasticoviscous fluid past an infinite wall with time-dependent suction, Acta Mech. 153 (2002), no. 3 4, 133-145.

[9] T. Hayat, S. Asghar, and A. M. Siddiqui, Periodic unsteady flows of a non-Newtonian fluid, Acta Mech. 131 (1998), no. 3-4, 169-175.

[10] On the moment of a plane disk in a non-Newtonian fluid, Acta Mech. 136 (1999), no. 3$4,125-131$.

[11] Some unsteady unidirectional flows of a non-Newtonian fluid, Internat. J. Engrg. Sci. 38 (2000), no. 3, 337-345.

[12] T. Hayat and K. Hutter, Rotating flow of a second-order fluid on a porous plate, Internat. J. NonLinear Mech. 39 (2004), no. 5, 767-777.

[13] T. Hayat, M. Khan, A. M. Siddiqui, and S. Asghar, Transient flows of a second grade fluid, Internat. J. Non-Linear Mech. 39 (2004), no. 10, 1621-1633. 


\section{Rotating second-grade flow with suction}

[14] P. N. Kaloni, Fluctuating flow of an elastico-viscous fluid past a porous flat plate, Phys. Fluids 10 (1967), no. 6, 1344-1346.

[15] R. E. Kelley, The flow of a viscous fluid past a wall of infinite extent with time-dependent suction, Quart. J. Mech. Appl. Math. 18 (1964), 287-298.

[16] M. J. Lighthill, The response of laminar skin friction and heat transfer to fluctuations in the stream velocity, Proc. Roy. Soc. London Ser. A 224 (1954), 1-23.

[17] I-C. Liu, Effect of modulation on onset of thermal convection of a second-grade fluid layer, Internat. J. Non-Linear Mech. 39 (2004), no. 10, 1647-1657.

[18] S. A. S. Messiha, Laminar boundary layers in oscillatory flow along an infinite flat plate with variable suction, Proc. Comb. Phil. Soc. 62 (1966), 329-337.

[19] K. R. Rajagopal, A note on unsteady unidirectional flows of a non-Newtonian fluid, Internat. J. Non-Linear Mech. 17 (1982), no. 5-6, 369-373.

[20] - Longitudinal and torsional oscillations of a rod in a non-Newtonian fluid, Acta Mech. 49 (1983), 281-285.

[21] On the creeping flow of the second-order fluid, J. Non-Newtonian Fluid Mech. 15 (1984), no. 2, 239-246.

[22] P. G. Siddheshwar and C. V. Sri Krishna, Unsteady non-linear convection in a second-order fluid, Internat. J. Non-Linear Mech. 37 (2002), no. 2, 321-330.

[23] V. M. Soundalgekar and P. Puri, On fluctuating flow of an elastico-viscous fluid past an infinite plate with variable suction, J. Fluid Mech. 35 (1969), 561-573.

[24] B. Straughan, Energy stability in the Bénard problem for a fluid of second grade, Z. Angew. Math. Phys. 34 (1983), no. 4, 502-509.

[25] J. T. Stuart, A solution of the Navier-Stokes and energy equations illustrating the response of skin friction and temperature of an infinite plate thermometer to fluctuations in the stream velocity, Proc. Roy. Soc. London Ser. A 231 (1955), 116-130.

T. Hayat: Department of Mathematics, Quaid-i-Azam University, 45320 Islamabad, Pakistan E-mail address: t_pensy@hotmail.com

Zaheer Abbas: Department of Mathematics, Quaid-i-Azam University, 45320 Islamabad, Pakistan E-mail address: za_qau@yahoo.com

S. Asghar: Department of Mathematics, Quaid-i-Azam University, 45320 Islamabad, Pakistan Current address: Department of Mathematical Sciences, COMSATS Institute of Information Technology, Plot no. 30, Sector H-8, Islamabad, Pakistan

E-mail address: s_asgharpk@yahoo.com 


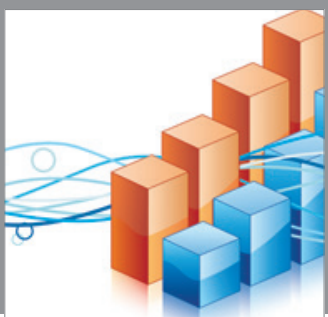

Advances in

Operations Research

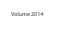

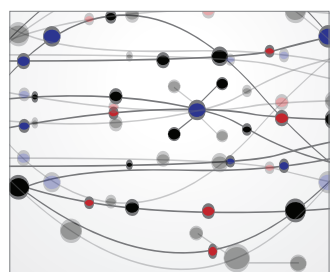

\section{The Scientific} World Journal
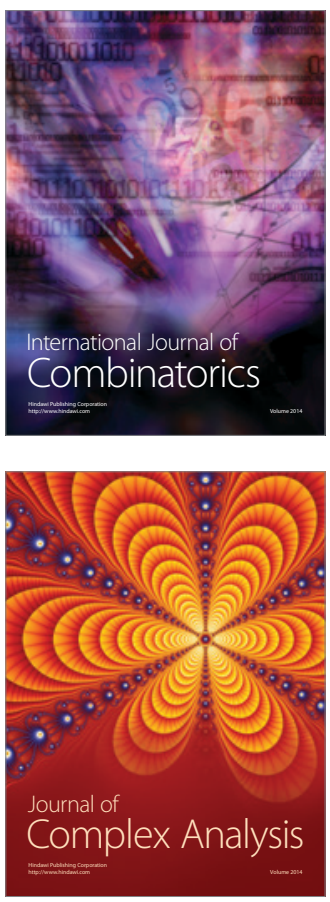

International Journal of

Mathematics and

Mathematical

Sciences
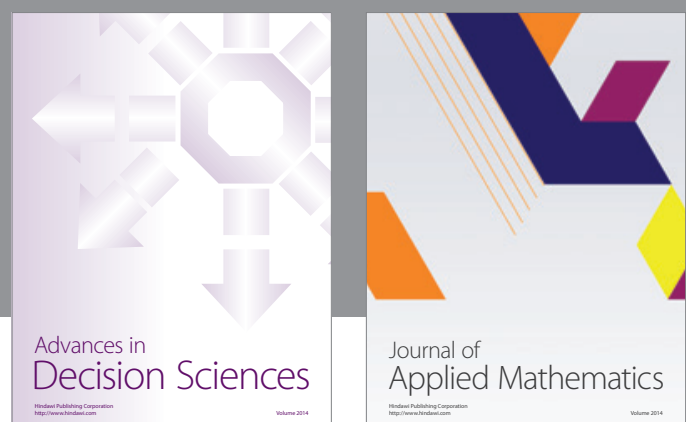

Journal of

Applied Mathematics
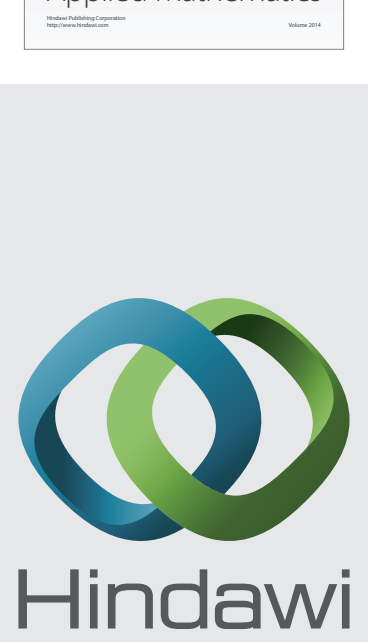

Submit your manuscripts at http://www.hindawi.com
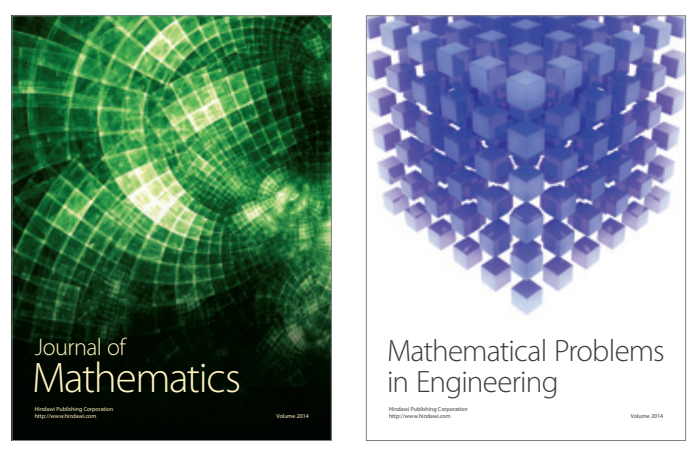

Mathematical Problems in Engineering
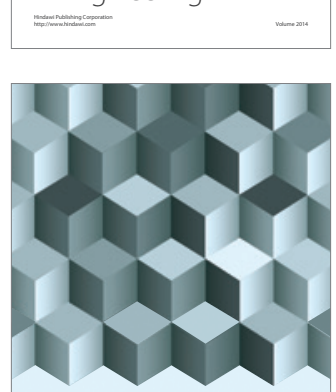

Journal of

Function Spaces
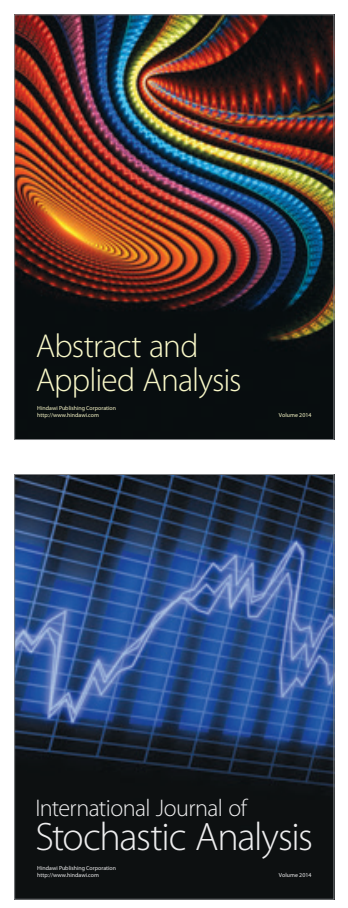

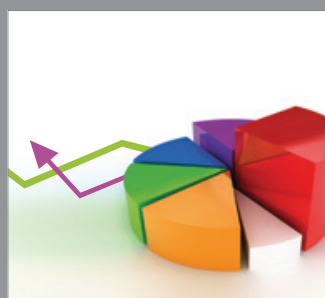

ournal of

Probability and Statistics

Promensencen
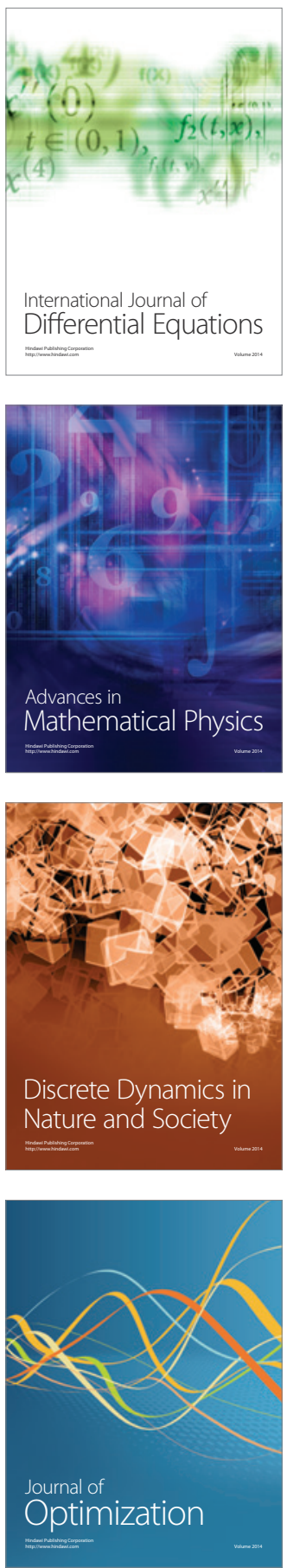